\title{
On Bulk Models of Shallow Cumulus Convection
}

\author{
GILLES BELLON \\ Laboratoire de Météorologie Dynamique du CNRS, IPSL, Université Pierre et Marie Curie, Paris, France \\ BJORN STEVENS \\ Department of Atmospheric Sciences, University of California, Los Angeles, Los Angeles, California
}

(Manuscript received 8 October 2003, in final form 22 September 2004)

\begin{abstract}
Two-layer bulk models of the cloudy boundary layer are investigated, focusing on the consistency issues between common assumptions on the turbulent mixing and the thermodynamic profiles. Simplifications of the vertical structure of the conserved variables are shown to lead to inconsistent steady states. First, linear profiles in the cloud layer are incompatible with the common parameterizations of the turbulent mixing. Second, this approximation is inconsistent with simple models of an infinitely thin inversion layer. Simple mass-flux parameterizations of the turbulence are shown to produce reasonable profiles inside the cloud layer without solving the problem of the inversion. The cloud - environment difference and the vertical gradient of the conserved variables appear as interesting test variables for such models, as well as their ability to match the free-tropospheric boundary conditions. The latter certainly requires a unified approach for the cloud and inversion layers.
\end{abstract}

\section{Introduction}

Processes that take place in the cloud-topped boundary layer $(\mathrm{CTBL})$ in the regions of the trade winds are important to the dynamics and thermodynamics of the tropical atmosphere as a whole. Not only does the depth of the boundary layer condition the low-level convergence of moist energy in the deep Tropics, which in turn helps determine the amount of deep convection, but the details of the vertical structure of the CTBL also determine its cloudiness, whose radiative forcing is an important feedback of the tropical system. While regions of boundary layer stratocumulus are thought to constitute a strong stabilizing feedback in the tropical atmosphere (Miller 1997), the radiative response of the trade wind regions is crucial to the sensitivity of the tropical climate (Bony et al. 2004). Through their modulation of the tropical climate, trade wind clouds regulate the climate as a whole. The variability among treatments of CTBL cloud processes is a leading explanation for the varied climate sensitivity of general circulation models (GCMs) noted in Cess et al. (1996).

Bulk models of the boundary layer are interesting tools to study the climate sensitivity (Betts and Ridg-

Corresponding author address: Gilles Bellon, Laboratoire de Météorologie Dynamique, Université Pierre et Marie Curie, case 99, 4 place Jussieu, 75252, Paris Cedex 5, France.

E-mail: Gilles.Bellon@lmd.jussieu.fr way 1989). Because they often encapsulate our understanding, bulk models also provide a convenient framework for evaluating parameterization ideas. The steady states of these models can give a good description of the bulk state of a real CTBL, or at least the bulk state to which it tends. Some one-layer models have proven their efficiency in representing the boundary layer. For instance, mixed-layer models (e.g., Lilly 1968) have allowed a better theoretical understanding of the dry convection and the stratocumulus-capped boundary layer.

Later, Betts and Ridgway $(1988,1989)$ developed the mixing-line model for the CTBL, which is based on empirical relationships describing the mixing of the air rising from the surface with the air descending from the free troposphere. Notwithstanding that a mixing line does not respect diabatic processes other than mixing, a limitation of this model is that the cloud-layer structure is slaved to the subcloud layer through a fixed parameter $\beta$ that depends on the nature (cloudy or clear sky) of the profile. Thus, the degrees of freedom exercised by the model are equivalent to that of a single-layer model. Because of these limitations, it is interesting to explore two-layer models, or equivalent two-layer models that truly allow extra degrees of freedom in the cloud layer and typically require additional closure assumptions. In principle, these models should allow a more realistic treatment of the variability in the vertical structure of the cloud layer, thereby improving the representation of cloudiness, and cloud depth within the 


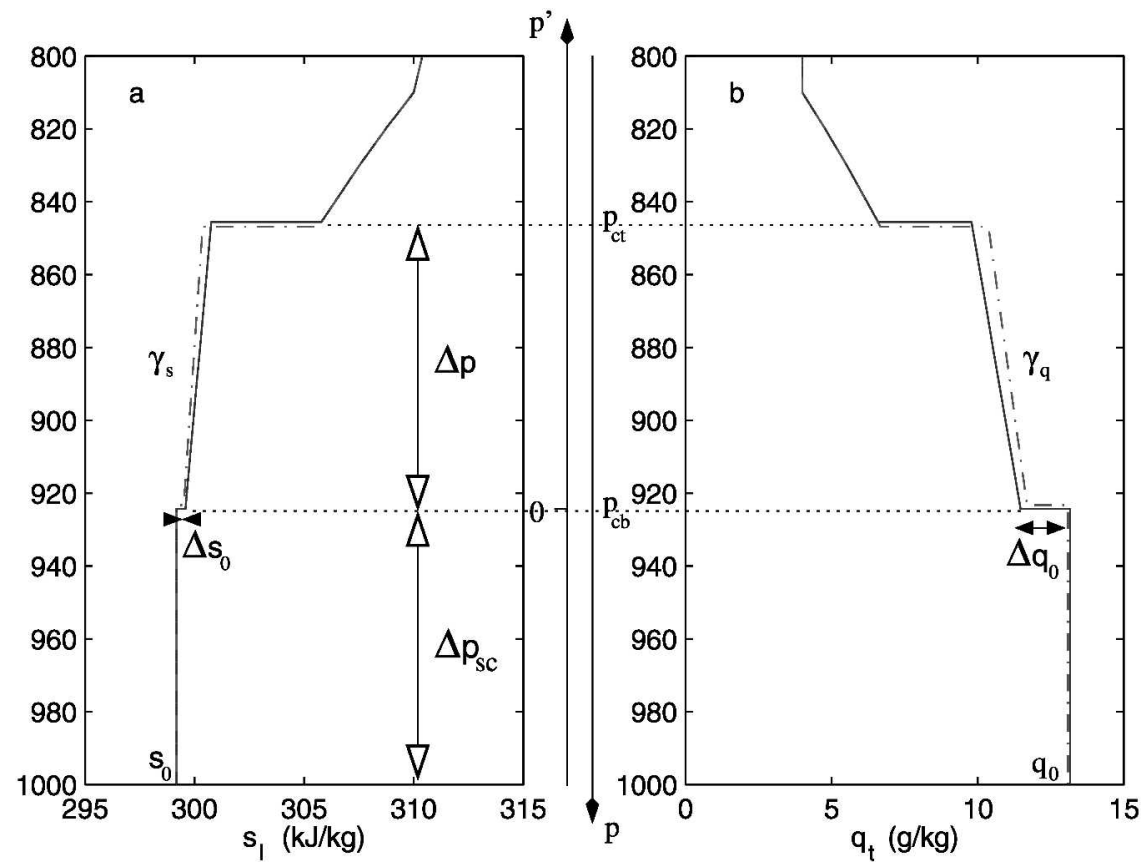

FIG. 1. Profiles of (a) liquid static energy and (b) total water produced by Albrecht model; solid line: Albrecht model; dash-dotted line: Albrecht model without linearization of the cloud - environment difference.

CTBL, both of which are critical from the larger-scale perspective. In so far as the additional closure assumptions are concerned, these usually take the form of a specification of a mean-field representation of the turbulent fluxes. In large part, it is thought that the fidelity of this representation determines the overall degree to which variability in the height and vertical structure of the CTBL are better represented in two-layer models.

The best-known example of a two-layer model of the CTBL is the one developed by Albrecht et al. (1979, hereafter A79) and Albrecht (1984, hereafter A84). This model consists of a well-mixed subcloud layer capped by a cloud layer in which the profiles of the conserved variables are linear and the turbulence is parameterized by a mass-flux scheme. It has been criticized in past work because of its sensitivity to poorly constrained parameters (Bretherton 1993). More recent work calls into question its closure assumptions: the turbulence parameters produced by large eddy simulation (LES) models (Siebesma and Cuijpers 1995, hereafter SC95; Stevens et al. 2001) and used in recent parameterizations (Siebesma and Holtslag 1996, hereafter SH96) are an order of magnitude bigger than the ones produced by the Albrecht model. This raises the question: Do improved models of cloud mixing lessen the sensitivity of the model to tunable parameters? Indeed, to what extent can recent advances in our understanding of the boundary layer improve our theoretical description at a bulk level?

The present work is devoted to answering these questions. We begin by reviewing the Albrecht model in section 2. Section 3 examines the relationship between the popular models of the cloud layer fluxes and the assumed cloud layer structure. Section 4 discusses the compatibility of Albrecht's framework with more general formulations of the turbulence, and section 5 examines the prospects of two-layer models with more degrees of freedom, with the idea of developing insight into remaining challenges confronting attempts to develop consistent bulk theories of the structure of the trade wind boundary layer.

\section{A two-layer framework}

\section{a. Thermodynamic structure}

The shallow cumulus boundary layer can be represented by two idealized layers, as in Albrecht's model (Fig. 1): (i) a well-mixed subcloud layer; (ii) a cloudy layer where average vertical gradients $\gamma_{q}$ and $\gamma_{s}$ of the conserved variables (i.e., the total water ratio $q_{t}=q+$ $q_{l}$ and the static liquid energy $\left.s_{l}=c_{p} T+g z-L q_{l}\right)$ are independent of height. This assumption will be hereafter called "constant gradient" hypothesis. (iii) The interface between the two layers, as well as the inversion layer, is characterized by jumps of humidity and temperature. These interfaces are considered here as infinitely thin and represented by the jumps $\Delta q_{0}$ and $\Delta s_{0}$. Such profiles can be described as follows:

$$
\begin{gathered}
p>p_{\mathrm{cb}} \quad \begin{aligned}
q_{t}(p) & =q_{0} \quad s_{l}(p)=s_{0}
\end{aligned} \\
p_{\mathrm{cb}}>p>p_{\mathrm{ct}} \quad\left\{\begin{array}{l}
q_{t}(p)=q_{0}+\Delta q_{0}+\gamma_{q} p^{\prime}, \\
s_{l}(p)=s_{0}+\Delta s_{0}+\gamma_{s} p^{\prime}
\end{array}\right.
\end{gathered}
$$


where $p$ is the pressure, the subscript ${ }_{\mathrm{cb}}\left({ }_{\mathrm{ct}}\right)$ indicates the cloud base (cloud top); $p^{\prime}=p_{\mathrm{cb}}-p$ is the pressure difference with the cloud base, which will be hereafter used as a vertical coordinate. Note that, following Albrecht, $p^{\prime}$ increases with height.

\section{b. Large-scale conditions}

Locally, we are looking at how the state of the equilibrium boundary layer depends on the large-scale conditions as measured by the sea surface state, surface divergence $D$, and radiative forcing.

The latter consists of two components: a clear-sky component $R$ and a cloud component $\Delta F^{R}$ that depends on the cloud fraction and the optical depth of the clouds. We assume that $R$ is homogeneous. Following recent work (SH96; Stevens et al. 2001), we further assume that the shallow cumuli cause no radiative forcing, whereas the stratiform clouds cause a cooling at the top of the boundary layer. The cloud radiative forcing can thus be approximated by a discontinuity $\Delta F^{R}$ of the net upward radiative flux at the cloud top:

$$
\Delta F^{R}=f_{s} \Delta F_{0}^{R},
$$

where $f_{s}$ is the stratiform cloud fraction and $\Delta F_{0}^{R}=$ $75 \mathrm{~W} \mathrm{~m}^{-2}$. It corresponds to a simplified version of the parameterization of the cloud radiative forcing in Stevens et al. (2001), with an average liquid water path of $3 \mathrm{~g} \mathrm{~m}^{-2}$ in the stratocumulus.

In the trade wind regions, the free troposphere varies on time scales of order days, which is long compared to the turbulent time scale of the CTBL (order tens of minutes). Thus, it makes sense to ask how the boundary layer structure develops as a function of some prescribed free-tropospheric state, which we specify as some linear function of $p^{\prime}$ according to

$$
\begin{aligned}
& q_{i+}=q_{i}^{o}+\Gamma_{q} p^{\prime}, \\
& s_{i+}=s_{i}^{o}+\Gamma_{s} p^{\prime},
\end{aligned}
$$

where the subscript ${ }_{i+}$ indicates the value at the top of the inversion, $\Gamma_{q}$ and $\Gamma_{s}$ are the free-tropospheric vertical gradients of humidity and static energy; $q_{i}^{o}$ and $s_{i}^{o}$ are the reference humidity and static energy at the cloud base. In our study, choosing the cloud base as a reference altitude is equivalent to choosing the surface or tropopause levels, and it simplifies the notations.

To maintain these profiles of the free-tropospheric thermodynamic structure, radiative cooling must be balanced by advection. Usually the balance is enforced only through the consideration of the vertical component of advection, called subsidence and denoted by $\omega$. In the case of a fixed gradient in $s_{l}$, a constant radiative cooling implies that $\omega=\omega_{i+}$ for $p \leq p_{\text {ct }}$. To have a positive rate of subsidence at, and above, the top of the CTBL, continuity demands that the large-scale divergence $D$ of the horizontal winds is positive within the CTBL. If we further consider that $D$ is constant with height within the CTBL then this is sufficient to completely specify a consistent vertical velocity

$$
\omega=D\left(p_{s}-p\right)=D\left(\Delta p_{\mathrm{sc}}+p^{\prime}\right),
$$

where $p_{s}$ is the surface pressure and $\Delta p_{\mathrm{sc}}=p_{s}-p_{\mathrm{cb}}$ is the thickness of the subcloud layer. Note that one drawback of this formulation is that because $p_{\text {ct }}$ is determined by the model, specifying $D$ does not guarantee that the subsidence at cloud top $\omega_{\mathrm{ct}}$ will adopt a value consistent with the subsidence $\omega_{i+}$ above the inversion.

\section{c. Fluxes and budgets: Additional information}

\section{1) Turbulent fluXes neEded to COMPute A CONSTANT GRADIENT MODEL}

The time-dependent equation of isentropic invariants (e.g., total water, moist entropy) can be written as

$$
\partial_{t} \phi=\omega \gamma_{\phi}-g \partial_{p^{\prime}} F^{\phi}+\sigma_{\phi} .
$$

Here, $\phi \in\left\{s_{l}, q_{t}\right\}$ denotes one of our thermodynamic state variables; $F^{\phi}$ the turbulent flux of $\phi$ and $\sigma_{\phi}$ is a source associated with irreversible processes acting on $\phi$, which for the most part we restrict to be independent of height below $p_{\text {ct }}$ such that $\sigma_{s_{l}}=R$ and $\sigma_{q t}=0$. As well, $F^{\phi}$ can be thought to include the fluxes due to precipitation, which are negligible at the surface but not at the cloud base. For a uniform diabatic source, the prognostic equation of the vertical gradient can be obtained by differentiating (1) with respect to $p^{\prime}$,

$$
\partial_{t} \gamma_{\phi}=D \gamma_{\phi}-g \partial_{p^{\prime} p^{\prime}} F^{\phi} .
$$

If we approximate the last term on the right-hand side (rhs) using divided differences, this expression can be written as follows:

$$
\partial_{t} \gamma_{\phi}=D \gamma_{\phi}-4 g \frac{F_{\mathrm{ct}}^{\phi}-2 F_{A}^{\phi}+F_{\mathrm{cb}}^{\phi}}{\Delta p^{2}},
$$

where $\Delta p$ is the depth of the cloud and subscript $A$ denotes the midpoint value, for example, $p_{A}=1 / 2\left(p_{\mathrm{ct}}+\right.$ $\left.p_{\mathrm{cb}}\right)$, or $p_{A}^{\prime}=1 / 2\left(p_{\mathrm{cb}}-p_{\mathrm{ct}}\right)=\Delta p / 2$. Because subcloud layer budgets give the time variation of the variables at the surface once the time evolution of $\gamma_{\phi}$ is known, the time evolution of the jump at cloud base can be derived using the budget of the full cloud layer. The latter involves the flux at cloud base and cloud top-both of which were required to specify $\partial_{t} \gamma_{\phi}$. The time evolution of the cloud layer, and its equilibrium, can thus be determined by a parameterization that specifies the fluxes at cloud base, cloud top, and at $p_{A}$ in terms of known quantities. It can be thought of as the closure hypothesis required for the two-layer model.

\section{2) Profiles of the fluXes in a steady state}

Assuming that the cloud layer can be in a steady state, and given the large-scale forcing, the turbulent 
fluxes can be written from (1) as a function of the vertical gradient $F^{\phi}=F^{\phi}\left(\gamma_{\phi}\right)$ :

$$
\partial_{p^{\prime}} F^{\phi}=\frac{D}{g}\left(\Delta p_{\mathrm{sc}}+p^{\prime}\right) \gamma_{\phi}+\frac{\sigma_{\phi}}{g},
$$

which, for constant $\gamma_{\phi}$, implies that the turbulent fluxes are quadratic in $p^{\prime}$ :

$$
F^{\phi}=F_{\mathrm{cb}}^{\phi}+\frac{D}{2 g}\left[\left(\Delta p_{\mathrm{sc}}+p^{\prime}\right)^{2}-\Delta p_{\mathrm{sc}}^{2}\right] \gamma_{\phi}+\frac{\sigma_{\phi}}{g} p^{\prime} .
$$

This is consistent with the fact the two-layer model requires the values of the flux at three levels in the cloud layer: a quadratic function can be entirely defined by its values at three different points.

\section{d. Parameterization of the mixing}

Albrecht's model, as well as more recent works (Tiedtke 1989; SH96), uses a mass-flux scheme to parameterize the turbulent fluxes that is based on a oneplume approach. This parameterization is often called "bulk mass flux" parameterization. It can be written as follows:

$$
\begin{aligned}
F^{\phi} & =M\left(\phi^{c}-\phi\right), \\
\partial_{p^{\prime}} M & =(\epsilon-\delta) M, \\
\partial_{p^{\prime}} \phi^{c} & =-\epsilon\left(\phi^{c}-\phi\right) .
\end{aligned}
$$

Here $\phi$ and $F^{\phi}$ retain their prior meaning except that the superscript ${ }^{c}$ indicates an expected value within the cloud; $M$ is the convective mass flux, $\epsilon$ and $\delta$ are the fractional entrainment and detrainment rates, respectively. In most of the current parameterizations (A79; A84; Tiedtke 1989; SH96), $\epsilon$ is independent of height, in which case (8) can then be integrated to obtain the cloud - environment difference

$$
\phi^{c}-\phi=\Delta \phi_{\mathrm{cb}} e^{-\epsilon p^{\prime}}-\frac{\gamma_{\phi}}{\epsilon}\left(1-e^{-\epsilon p^{\prime}}\right),
$$

where $\Delta \phi_{\mathrm{cb}}$ is the cloud - environment difference at the cloud base. In Albrecht's model, $\epsilon$ is parameterized as $E / \Delta p$, where $E$ is a parameter depending on the buoyancy profile [see Eqs. (35) and (36) in A79]. Equations (6) and (7) can also be written:

$$
\begin{aligned}
F^{\phi} & =F_{\mathrm{cb}}^{\phi} f\left(p^{\prime}\right) d\left(p^{\prime}, \zeta_{\phi}\right), \\
M & =M_{\mathrm{cb}} f\left(p^{\prime}\right),
\end{aligned}
$$

where $f$ is a function of the pressure that gives the evolution of the mass flux with height $[f(0)=1]$ and $d$ gives the evolution of the cloud - environment difference with height $[d(0)=1]$, which depends also on the ratio $\zeta_{\phi}=\gamma_{\phi} / \Delta \phi_{\mathrm{cb}}$ between the vertical gradient and the cloud - environment difference at cloud base:

$$
d\left(p^{\prime}, \zeta_{\phi}\right)=e^{-\epsilon p^{\prime}}-\frac{\zeta_{\phi}}{\epsilon}\left(1-e^{-\epsilon p^{\prime}}\right) .
$$

Any pair of functions $(\epsilon, \delta),(\epsilon, f)$, and $(f, d)$ uniquely describes the parameterization. Conversely, whatever the profiles of conserved variables may be, any pair of flux profiles $\left(F^{q}, F^{s}\right)$ can be parameterized by any pair of those functions: the mass-flux formalism per se does not place any constraint on the system.

On the contrary, common $K$ diffusion schemes do constrain the turbulent fluxes, since the mixing is described by only one function $K$ (Stevens 2000); in this type of scheme, the turbulent fluxes are parameterized as follows:

$$
F^{\phi}=-K \gamma_{\phi} .
$$

From Eq. (5), it is readily apparent that the $K$ diffusion scheme is inconsistent with the assumption of a constant-gradient steady state

$$
\frac{F^{\phi}}{\gamma_{\phi}}=\frac{F_{\mathrm{cb}}^{\phi}}{\gamma_{\phi}}+\frac{D}{2 g}\left[\left(\Delta p_{\mathrm{sc}}+p^{\prime}\right)^{2}-\Delta p_{\mathrm{sc}}^{2}\right]+\frac{\sigma_{\phi}}{g \gamma_{\phi}} p^{\prime},
$$

and it is unlikely to be independent of $\phi$ since it would require $F_{\mathrm{cb}}^{\phi} / \gamma_{\phi}$ and $\sigma_{\phi} / \gamma_{\phi}$ to be independent of $\phi$. Of course, using two different $K$ profiles is possible, although it takes more elaborate justification than the classical $K$ diffusion schemes.

It is straightforward to ask what assumptions on the functions describing a mass-flux scheme are compatible with the constant gradient hypothesis. To start with, we can study whether the parameterizations used in A79 or developed more recently (SH96) can lead to a steady state in such a model.

\section{Steady-state solutions?}

In this section and the next one, we will study how the steady-state solutions of the two-layer constant gradient model constrain the functions describing the mixing. For this purpose, we will assume that the whole boundary layer is in steady state and, focusing on the cloud and inversion layer, we will derive necessary conditions on the parameters of the turbulence or the characteristics of the inversion.

\section{a. Required values of the parameters}

Given a mass-flux parameterization as described in the previous section, we can assume the existence of a steady state of the constant gradient model, in which case, Eq. (5) requires that

$$
f\left(p^{\prime}\right) d\left(p^{\prime}, \zeta_{\phi}\right)=1+\frac{D}{2 g M_{\mathrm{cb}}}\left(p^{\prime 2}+2 p^{\prime} \Delta p_{\mathrm{sc}}\right) \zeta_{\phi}+\frac{\sigma_{\phi}}{g F_{\mathrm{cb}}^{\phi}} p^{\prime} .
$$

As we stated in section $2 \mathrm{c}$, the model can reach an equilibrium if (15) is verified at least at the top and the middle of the cloud. 
With those two conditions, we can eliminate the term involving the diabatic forcing and the flux at cloud base to obtain

$$
1-2 f(\Delta p / 2) d\left(\Delta p / 2, \zeta_{\phi}\right)+f(\Delta p) d\left(\Delta p, \zeta_{\phi}\right)=\frac{D \Delta p^{2}}{4 g M_{\mathrm{cb}}} \zeta_{\phi} .
$$

Equation (16) can also be derived from (3), for the steady state of the vertical gradient.

The expression of $d$ [Eq. (12)] shows that the lefthand side (lhs) of (16) is linear in $\zeta_{\phi}$. For Eq. (16) to be verified for total water and static energy, the following condition is necessary:

$$
1-2 f(\Delta p / 2) e^{-\epsilon \Delta p / 2}+f(\Delta p) e^{-\epsilon \Delta p}=0 .
$$

Note that this equation is an expression of the interplay between the fluxes and profiles, and thus depends not at all on the bulk budget, but only on the assumptions on the profiles and in the parameterization of the mixing. Equation (17) always yields to the trivial solution $\Delta p=0$, which reduces the model to a well-mixed onelayer model. We cannot describe the other solutions without specifying the function $f$. We will analyze two closures that have seemed to be the most reasonable approximations and have been used for two-layer or one-dimensional (1D) models.

In Albrecht's model, the convective mass flux is supposed to vary linearly with height: $f\left(p^{\prime}\right)=1+\mu p^{\prime}$. Equation (17) can be rewritten:

$$
\left(1-e^{-\epsilon \Delta p / 2}\right)\left[1-e^{-\epsilon \Delta p / 2}(1+\mu \Delta p)\right]=0,
$$

which yields to either (i) a mass flux strictly increasing with height

$$
\mu=\frac{e^{\epsilon \Delta p / 2}-1}{\Delta p}>0,
$$

or (ii) no entrainment: $\epsilon=0$.

In SH96, it is suggested that the fractional detrainment rate $\delta$ is independent of height, as well as $\epsilon$; the mass flux decreases exponentially: $f\left(p^{\prime}\right)=e^{(\epsilon-\delta) p^{\prime}}$. Equation (17) turns into

$$
\left(1-e^{-\delta \Delta p / 2}\right)^{2}=0
$$

which yields to no detrainment $\delta=0$. The convective mass flux increases strictly with height if the entrainment is not also zero.

Though an increase of the mass flux can be observed in some cases near cloud base or cloud top (Stevens et al. 2001), the monotonic increase of the convective mass flux is far from being the general case. The alternative absence of entrainment is also unrealistic in shallow cumuli (SC95; Stevens et al. 2001; Blyth 1993): even the air in the cloud cores gets somewhat diluted by air from the environment. Thus, the linear profiles seem inconsistent with a bulk mass-flux parameterization of the turbulent fluxes that uses a constant fractional en- trainment: the model cannot reach an equilibrium with such a parameterization.

Here, we can see that the constraints on the profiles of the conserved variables can affect the mixing in a way that keeps the system unsteady. In general, the assumptions about the nature of the mixing determine the equilibrium profile. Similarly, assumptions about the state determine, or at least constrain, the mixing. This raises the question of why Albrecht's model "works."

\section{b. Why does Albrecht's model work?}

\section{1) Albrecht's simplificAtions of THE PARAMETERIZATION}

In A79, an Atlantic Trade Winds Experiment (ATEX) case is successfully reproduced. However, different mathematical approximations are used in A79 and A84 to simplify the mass flux scheme. Some of them prove to have a decisive impact on the equilibrium of the model. (i) The cloud - environment differences $\left(\phi^{c}-\phi\right)$ of the conserved variables $q_{t}$ and $s_{l}$ are linearized. (ii) The flux $F_{\mathrm{ct}}^{q}$ of water at the top of the cloud is constrained to respect the budget of the thin inversion layer.

We reproduce here the base case of A79 except for the closure on $E$ that is taken from A84 (it corresponds to the A84 model without horizontal advection). The equilibrium profiles are shown in Fig. 1. To analyze the impact of the simplifications, we release each of those constraints.

\section{2) LINEARIZATION OF THE PROFILES}

In Albrecht's model, the cloud - environment differences given by Eq. (9) are linearized constraining the cloud base values and the total amount of water/liquid static energy in the cloud layer. Note that the approximated cloudy profiles do not verify Eq. (8). If we release that approximation and use the profiles given by (9), the equilibrium total water and temperature profiles are close to the base case (see Fig. 1). However, the flux profiles are quite different, the convective mass flux increases with the altitude as stated in section $3 \mathrm{a}$ (Fig. 2a), and that leads to bigger heat and moisture fluxes at the top of the cloud (Figs. $2 b$ and $2 c$ ).

But the model uses only the water flux at the cloud base, in the middle of the cloud and at the top of the cloud (indicated by circles and triangles on the profile in Fig. 2c). The water flux at the top of the cloud is prescribed by the budget of the inversion layer [constraint (ii)] and this approximation allows the model to find an equilibrium somewhat independently from the mass-flux parameterization.

\section{3) Flux of WAter at THE TOP OF THE ClOUd}

If we release the constraint on the cloud-top water flux (allowing the budget of water in the inversion layer 

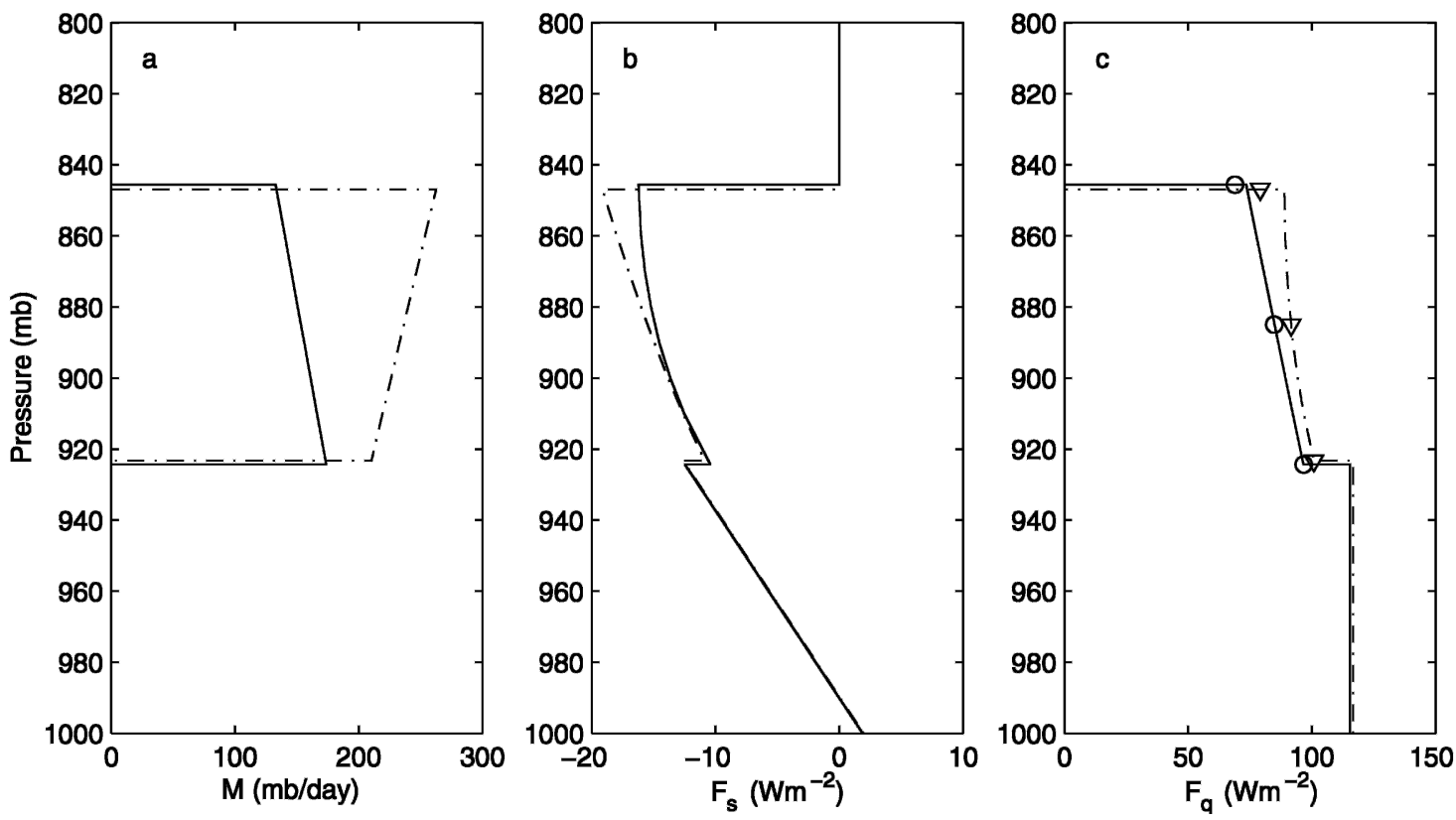

FIG. 2. Profiles of (a) convective mass flux, (b) turbulent heat flux, and (c) turbulent moisture flux; same conventions as previously, plus circles (triangles) represent moisture fluxes that are actually used in Albrecht model (Albrecht model without linearization).

not to be verified), the modeled boundary layer dries up to become dry and well mixed: the cloud layer disappears $(\Delta p=0$ as stated in section 3a).

In the absence of cloud radiative forcing $\left(\Delta F^{R}=0\right.$, which corresponds to the BOMEX case), the residual $r_{\mathrm{ct}}^{q}$ between the flux of water prescribed by the balance of the inversion layer and $F_{\mathrm{ct}}^{q}$ computed by the parameterization of the mixing can be written as

$$
r_{\mathrm{ct}}^{q}=F_{\mathrm{ct}}^{S}\left(\frac{q_{i+}-q_{\mathrm{ct}}}{S_{i+}-S_{\mathrm{ct}}}-\frac{q_{\mathrm{ct}}^{c}-q_{\mathrm{ct}}}{S_{\mathrm{ct}}^{c}-S_{\mathrm{ct}}}\right) .
$$

This residual flux vanishes if the cloud - environment differences at cloud top, normalized by the jumps across the inversion, are equal for both conserved variables. This is actually a strong boundary condition for the parameterization of the turbulent fluxes. As shown by the circle at cloud top in Fig. 2, this residual happens to be small in the ATEX case when the cloud - environment difference is linearized, but it is larger when this mathematical approximation is not applied (see the triangle in Fig. 2).

The equilibrium of Albrecht model relies on a mathematical approximation and on an arbitrary constraint that modify the mass-flux scheme. Although these might seem like arcane points, they are critical. The consistency of the theoretical framework allows for a more critical evaluation of the effect of various assumptions on the cloud layer. This weakens Albrecht model's physical pertinence and might explain why its behavior depends so much on the tunable parameters (Bretherton 1993).

\section{Relaxed flux laws}

Parameterizations of the mixing with strong constraints on the flux profiles (constant entrainment, linear, or exponential mass flux) fail to represent a constant gradient cloud layer in steady state, even if they are applied only at three levels. Here we relax these constraints to investigate if there exist some form of $f$ and $d$ that allows a constant gradient cloud layer capped by an infinitely thin inversion to reproduce the observations. In the most general sense, we are exploring the compatibility of the mass-flux equations with the assumption of a constant gradient cloud layer.

To address this issue we consider the cloud-base altitude and fluxes as parameters that we take from both LES results (SC95; Stevens et al. 2001) and observations (Holland and Rasmusson 1973; Augstein et al. 1973): we further consider that the subcloud layer is in steady state and that the fluxes at cloud base are constrained by the balance of the subcloud layer. The necessary conditions derived from the equilibrium equations will be evaluated for conditions observed during the Barbados Oceanographic and Meteorological Experiment (BOMEX; Holland and Rasmusson 1973) and ATEX (Augstein et al. 1973). The observed altitude of the cloud top will also be used in the first subsection to bound certain terms in the resultant equations.

\section{a. Within the cloud: The constant gradient hypothesis in question}

If we assume that a constant gradient model can reach an equilibrium with more elaborate functions de- 
scribing the detrainment and entrainment from the clouds, we can build the functions $\epsilon\left(p^{\prime}\right)$ and $\delta\left(p^{\prime}\right)$ from Eqs. (6) to (8) as well as the mass-flux profile $M\left(p^{\prime}\right)$. Details of the derivation are given in appendix A. Here $M\left(p^{\prime}\right), \epsilon\left(p^{\prime}\right)$, and $\delta\left(p^{\prime}\right)$ can be expressed as a function of the turbulent fluxes and their derivatives and the vertical gradients of $s_{l}$ and $q_{t}$ :

$$
\begin{gathered}
M=-\frac{\frac{\partial_{p^{\prime}} F^{q}}{F^{q}}-\frac{\partial_{p^{\prime}} F^{s}}{F^{s}}}{\frac{\gamma_{q}}{F^{q}}-\frac{\gamma_{s}}{F^{s}}}, \\
\delta=-\frac{\frac{\partial_{p^{\prime}} F^{q}}{\gamma_{q}}-\frac{\partial_{p^{\prime}} F^{s}}{\gamma_{s}}}{\frac{F^{q}}{\gamma_{q}}-\frac{F^{s}}{\gamma_{s}}}, \\
\quad \frac{\partial_{p^{\prime} p^{\prime}} F^{q}}{F^{q}}-\frac{\partial_{p^{\prime} p^{\prime}} F^{s}}{F^{s}} \\
\frac{\partial_{p^{\prime}} F^{q}}{F^{q}}-\frac{\partial_{p^{\prime}} F^{s}}{F^{s}}
\end{gathered}
$$

The physical consistency of the mass-flux scheme demands $M>0, \epsilon>0$, and $\delta>0$. These constraints reduce the domain of equilibria that can be represented in the constant gradient framework for a given largescale forcing and fluxes at the cloud base. Using the expression of the fluxes at equilibrium [Eq. (5)], the domain of possible equilibrium $\left(\gamma_{q}, \gamma_{s}\right)$ can be bounded (see the appendix A for details):

$$
\begin{aligned}
& \gamma_{q} \beta_{F}(1+\alpha \pi)<\gamma_{s}<-\frac{R}{\omega_{\mathrm{ct}}}+\gamma_{q} \beta_{F}\left[1+\frac{\alpha \pi^{2}}{2(1+\pi)}\right] \\
& \gamma_{s}<\gamma_{q} \beta_{F}\left[1+\alpha\left(\sqrt{1-2 \frac{q_{\mathrm{cb}}^{e}}{\gamma_{q} \Delta p_{\mathrm{sc}}}}-1\right)\right]
\end{aligned}
$$

where $\pi=\Delta p / \Delta p_{\mathrm{sc}}$ is the ratio of the cloud depth to the subcloud-layer depth, $\beta_{F}$ is the ratio of the sensible to latent heat fluxes at the cloud base (the equivalent of the Bowen ratio at the cloud base) multiplied by $L, \alpha$ is the ratio of the total radiative cooling within the subcloud layer to the warming of this layer by the turbulent flux at cloud base, and $q_{\mathrm{cb}}^{e}$ is the equivalent humidity of the turbulent transport at the cloud base:

$$
\beta_{F}=\frac{F_{\mathrm{cb}}^{s}}{F_{\mathrm{cb}}^{q}}, \alpha=\frac{R \Delta p_{\mathrm{sc}}}{g F_{\mathrm{cb}}^{s}}, q_{\mathrm{cb}}^{e}=g \frac{F_{\mathrm{cb}}^{q}}{\omega_{\mathrm{cb}}} .
$$

Furthermore, we can analyze the change of the convective mass flux with height, a decrease of the mass flux with height corresponds to $\epsilon-\delta<0$. Using Eqs. (22) and (23), we can write

$$
\epsilon-\delta=\frac{\frac{\partial_{p^{\prime} p^{\prime}} F^{q}}{F^{q}}-\frac{\partial_{p^{\prime} p^{\prime}} F^{s}}{F^{s}}}{\frac{\partial_{p^{\prime}} F^{q}}{F^{q}}-\frac{\partial_{p^{\prime}} F^{s}}{F^{s}}}-\frac{\frac{\partial_{p^{\prime}} F^{q}}{\gamma_{q}}-\frac{\partial_{p^{\prime}} F^{s}}{\gamma_{s}}}{\frac{F^{q}}{\gamma_{q}}-\frac{F^{s}}{\gamma_{s}}} .
$$

In the common range of forcing, Eq. (26) yields to both the condition for a strictly decreasing mass flux (see appendix A for details):

$$
\begin{gathered}
\gamma_{s}>\gamma_{q} \beta_{F}\left[1+\frac{\alpha}{2}\left(\sqrt{1-2 \pi-\pi^{2}-4 \frac{q_{\mathrm{cb}}^{e}}{\gamma_{q} \Delta p_{\mathrm{sc}}}}\right.\right. \\
-1+\pi)]
\end{gathered}
$$

and the condition for a strictly increasing mass flux

$$
\gamma_{s}<\gamma_{q} \beta_{F}\left[1+\frac{\alpha}{2}\left(\sqrt{1-4 \frac{q_{\mathrm{cb}}^{e}}{\gamma_{q} \Delta p_{\mathrm{sc}}}}-1\right)\right] .
$$

Figure 3 shows the domains of potentially represented steady states, in the ATEX and BOMEX cases, using the large-scale forcings, boundary fluxes, and parameters indicated in Table 1 . The observations are partly out of the domain: the BOMEX case seems impossible to reproduce. Moreover, in the cases that can be reproduced, a decrease of the convective mass flux is a marginal case, whereas it is the most common case in the observations. In the ATEX case, a constant gradient model would produce a mass flux that would strictly increase with height, consistent with the results of section 3a. This appears unrealistic: though the convective mass flux increases at the top of the cloud layer because of stratiform clouds (Stevens et al. 2001), it decreases within most of the layer.

The increase of the mass flux with height is a direct consequence of an underestimation of the cloud - environment difference at the top of the cloud, which is due to the constant gradient and appears clearly in (9): this difference tends asymptotically toward $-\gamma_{\phi} / \epsilon$. This limit in the growth of the cloud - environment difference with height requires an increase of the convective mass flux with altitude to produce the equilibrium fluxes.

The increase of the convective mass flux with height is also characteristic of some parameterizations that are used in operational models, as noted in Lenderink et al. (2005); although it certainly helps represent more steady-state oceanic cases, Lenderink et al.'s work shows that it is one of the main problems in the simulation of the diurnal cycle over the continents.

Our result shows that the simple constant gradient framework can reproduce at best very few observed profiles when it is coupled to a bulk mass-flux scheme, whatever the entrainment and detrainment profiles might be: this type of parameterization depends too 

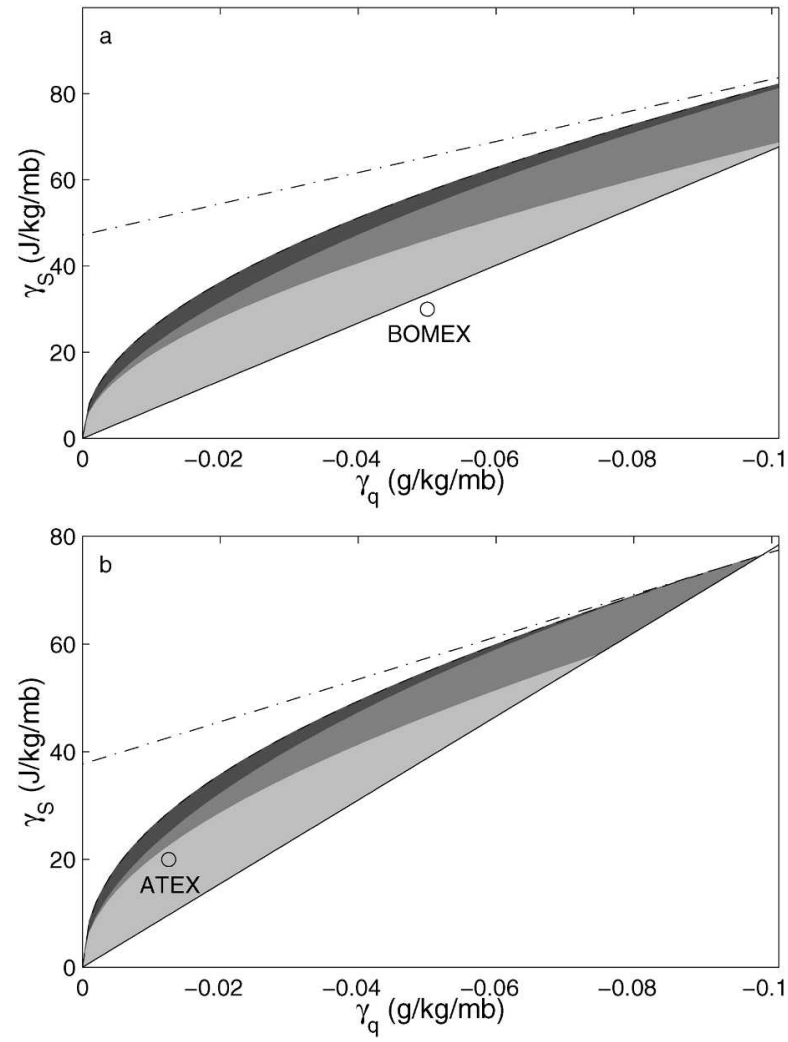

FIG. 3. Domains of equilibrium $\left(\gamma_{q}, \gamma_{s}\right)$ (shaded) that can be simulated by a bulk mass flux parameterization with (a) BOMEX and (b) ATEX large-scale forcing. Darkest (lightest) shade: mass flux strictly decreasing (increasing) with height; intermediary shade: nonmonotonic mass flux; the limits of the different shade zones are given by (27) and (28). Solid: lhs of (24); dash-dotted: rhs of (24); dashed: rhs of (25); the circles indicate the observed values.

much on the details of higher-order derivatives of the conserved variables to be used in such a simple framework. Part of the problem might result from our simplifications to the large-scale forcing or our neglect of transient or advective effects; nonetheless, it illustrates the strength of the physical constraints given by a oneplume scheme. In general, it seems that a constant gradient is essentially incompatible with the mass-flux formalism.
TABLE 1. Large-scale forcing and parameters used in computations.

\begin{tabular}{lcc}
\hline \hline Case & BOMEX & ATEX \\
\hline$D$ & $3 \times 10^{-6} \mathrm{~s}^{-1}$ & $5 \times 10^{-6} \mathrm{~s}^{-1}$ \\
$R$ & $-2 \mathrm{~K} \mathrm{day}^{-1}$ & $-2.5 \mathrm{~K} \mathrm{day}^{-1}$ \\
$f_{s}$ & 0.0 & 0.5 \\
$p_{s}$ & $1010 \mathrm{mb}$ & $1010 \mathrm{mb}$ \\
$P_{\mathrm{cb}}$ & $950 \mathrm{mb}$ & $930 \mathrm{mb}$ \\
$P_{c t}^{c}$ & $845 \mathrm{mb}$ & $855 \mathrm{mb}$ \\
$F_{\mathrm{cb}}^{q}$ & $125 \mathrm{~W} \mathrm{~m}^{-2}$ & $100 \mathrm{~W} \mathrm{~m}^{-2}$ \\
$F_{\mathrm{cb}}^{s}$ & $-12 \mathrm{~W} \mathrm{~m}^{-2}$ & $-12 \mathrm{~W} \mathrm{~m}^{-2}$ \\
\hline
\end{tabular}

\section{b. At the top of the cloud: The overconstrained massless inversion}

\section{1) THE INFINITELY THIN INVERSION}

In Albrecht's framework, the constant gradient hypothesis is a strong constraint on the profiles. The assumption of an infinitely thin inversion layer seems less questionable since it has proven so useful in the representation of the stratocumulus-topped boundary layer. Considering that some undetermined parameterization of the turbulence can produce an equilibrium cloud layer, we can investigate to what extent this assumption is compatible with the constant gradient hypothesis.

Taking the time derivative of $\phi$ integrated over a thin inversion layer around $p_{\mathrm{ct}}$ and taking the limit where the depth of this layer is zero, the budget of a conserved variable for the inversion can be written as follows:

$$
\left(\partial_{t} p_{\mathrm{ct}}^{\prime}+\omega_{\mathrm{ct}}\right)\left(\phi_{i+}-\phi_{\mathrm{ct}}\right)+g\left(F_{\mathrm{ct}}^{\phi}+\Sigma_{\phi}\right)=0,
$$

where $\Sigma_{\phi}$ is the diabatic source of $\phi$ within the inversion layer $\left(\Sigma_{q t}=0\right.$ and $\left.\Sigma_{s l}=-\Delta F^{R}\right)$.

The balance of static energy and total water of the infinitely thin inversion layer can thus be written

$$
\frac{F_{\mathrm{ct}}^{q}}{q_{i+}-q_{\mathrm{ct}}}=-\frac{\omega_{\mathrm{ct}}}{g}=\frac{F_{\mathrm{ct}}^{s}-\Delta F^{R}}{s_{i+}-s_{\mathrm{ct}}}
$$

with $q_{i+}$ and $s_{i+}$ assumed to vary linearly with the altitude of the cloud top, as stated in section $2 \mathrm{~b}$.

Assuming an equilibrium of the system with an adequate parameterization of the turbulent fluxes, $F_{\mathrm{ct}}^{q}$ and $F_{\mathrm{ct}}^{s}$ are given by Eq. (5). Knowing the vertical gradients $\gamma_{s}$ and $\gamma_{q}$, each part of (30) allows one to compute independently the thickness of the cloud layer $\Delta p$ :

$$
\begin{aligned}
& \Delta p=\frac{\Delta q_{i}^{o}+\Gamma_{q} \Delta p_{\mathrm{sc}}}{\gamma_{q}-2 \Gamma_{q}}\left(1-\sqrt{1-\frac{4\left(\Gamma_{q}-\frac{\gamma_{q}}{2}\right) \Delta p_{\mathrm{sc}}}{\left(\Delta q_{i}^{o}+\Gamma_{q} \Delta p_{\mathrm{sc}}\right)^{2}}\left(\Delta q_{i}^{o}+q_{\mathrm{cb}}^{e}\right)}\right), \\
& \Delta p=\frac{\Delta s_{i}^{o}+\Gamma_{s} \Delta p_{\mathrm{sc}}+\frac{R}{D}}{\gamma_{s}-2 \Gamma_{s}}\left(1-\sqrt{1-\frac{4\left(\Gamma_{s}-\frac{\gamma_{s}}{2}\right) \Delta p_{\mathrm{sc}}}{\left(\Delta s_{i}^{o}+\Gamma_{s} \Delta p_{\mathrm{sc}}+\frac{R}{D}\right)^{2}}\left(\Delta s_{i}^{o}+s_{\mathrm{cb}}^{e}\right)}\right),
\end{aligned}
$$


TABLE 2. Free-tropospheric profiles used in computations.

\begin{tabular}{lccccc}
\hline \hline Case & $q_{i}^{o}\left(\mathrm{~g} \mathrm{~kg}^{-1}\right)$ & $\Gamma_{q}\left(\mathrm{~g} \mathrm{~kg}^{-1} \mathrm{mb}^{-1}\right)$ & $s_{i}^{o}\left(\mathrm{~kJ} \mathrm{~kg}^{-1}\right)$ & $\Gamma_{s}\left(\mathrm{~kJ} \mathrm{~kg}^{-1} \mathrm{mb}^{-1}\right)$ & $\omega_{i}\left(\mathrm{mb} \mathrm{day}^{-1}\right)$ \\
\hline BOMEX & 6.0 & -0.01 & 306.6 & 30 & 35 \\
ATEX & 4.0 & 0.0 & 306.4 & 23 & 55 \\
\hline
\end{tabular}

where $s_{\mathrm{cb}}^{e}$ is the equivalent static energy of the turbulent transport at the cloud base, and $\Delta \phi_{i}^{o}$ is the temperature difference between the cloud layer profile and the freetropospheric profile at cloud base;

$$
s_{\mathrm{cb}}^{e}=g \frac{F_{\mathrm{cb}}^{s}-\Delta F^{R}}{\omega_{\mathrm{cb}}}, \Delta \phi_{i}^{o}=\phi_{i}^{o}-\phi_{0}-\Delta \phi_{0}
$$

This double equation for $\Delta p$ is a potential source of inconsistency for the framework and shows that the system is overconstrained. Recall that, in Albrecht's model, the total water flux at cloud top is not parameterized but computed in order to respect (30); this allows one to get a unique determination of $\Delta p$ with the energy budget of the inversion layer. A unique determination of $\Delta p$ requires the rhs of (31) to equal the rhs of (32). Given the large-scale conditions and the flux and variables at the cloud base, this equality leads to a constraint on $\left(\gamma_{q}, \gamma_{s}\right)$. Using the observed variables at the cloud base $\left(q_{\mathrm{cb}}, s_{\mathrm{cb}}\right)=\left(16 \mathrm{~g} \mathrm{~kg}^{-1}, 302 \mathrm{~kJ} \mathrm{~kg}^{-1}\right)$ and the parameters listed in Tables 1 and 2 for BOMEX, the possible equilibrium pairs $\left(\gamma_{q}, \gamma_{s}\right)$ are shown in Fig. 4 (solid line). The observed gradients do not seem possible to reproduce. Furthermore, Fig. 4 shows the equilibrium altitude of the cloud top along the line of possible equilibrium (dots and labels): gradients $\left(\gamma_{q}, \gamma_{s}\right)$ that are close to the observed ones allow the representation of an inversion between 700 and $500 \mathrm{mb}$, which is much higher than the observed cloud top around 850 $\mathrm{mb}$. One could argue that, by suitably choosing the fluxes at the cloud base, a reasonable steady state could be found (see dash-dotted line in Fig. 4). However, because these fluxes are constrained by the surface fluxes and the equilibrium of the subcloud layer, it is reasonable to consider them as parameters. Additionally, the gradients and the altitude of the inversion are not too sensitive to changes in these parameters: to reproduce the observed cloud layer, the dash-dotted line in Fig. 4 was computed with parameters that have been significantly modified:

$$
\left(F_{\mathrm{cb}}^{q}, F_{\mathrm{cb}}^{s}\right)=\left(100 \mathrm{~W} \mathrm{~m}^{-2},-4 \mathrm{~W} \mathrm{~m}^{-2}\right) .
$$

We do not need to perform the same computation for ATEX, Albrecht's model showed that it is possible to reproduce this case with a constant gradient model and an infinitely thin inversion layer using a truncated massflux parameterization. Our results show that it is impossible to model BOMEX case. This is consistent with the observations: the inversion was much less abrupt during BOMEX than it was during ATEX, thus it is less likely to be satisfactorily modeled by an infinitely thin layer.

\section{2) A MODEL FOR A THIN FINITE INVERSION}

Nevertheless, the model of infinitely thin inversion layer can be simply modified. Let's consider a thin inversion between $p_{\mathrm{ct}}$ and $p_{i+}$ where the profiles of conserved variables inside the inversion layer follow a mixing line. This model is slightly more general than an inversion layer with linear profiles. The balance of the layer for a conserved variable $\phi$ can be written as illustrated in Fig. 5:

$$
\omega_{i+} \phi_{i+}-\left(\omega_{i+}-\omega_{\mathrm{ct}}\right) \bar{\phi}-\omega_{\mathrm{ct}} \phi_{\mathrm{ct}}+g F_{\mathrm{ct}}^{\phi}+\Sigma_{\phi}=0,
$$

where $\Sigma_{\phi}$ is the diabatic source for the whole layer; $\bar{\phi}=$ $\lambda \phi_{i+}+(1-\lambda) \phi_{\mathrm{ct}}$ is the vertical average of $\phi$ in the layer; and $\lambda$ is a mixing coefficient between 0 and 1 that, since the profiles follow a mixing line, does not depend on $\phi$. For linear profiles, $\lambda=1 / 2$.

Assuming that the layer is sufficiently thin to neglect the effect of the variation of $\phi_{i+}$ between $p_{i+}$ and $p_{\text {ct }}$, Eq. (33) can be written for $q_{t}$ and $s_{l}$ to yield to the following equivalent of (30):

$$
\frac{F_{\mathrm{ct}}^{q}}{q_{i+}-q_{\mathrm{ct}}}=-\frac{\bar{\omega}}{g}=\frac{F_{\mathrm{ct}}^{s}-\Delta F^{R}}{s_{i+}-s_{\mathrm{ct}}}
$$

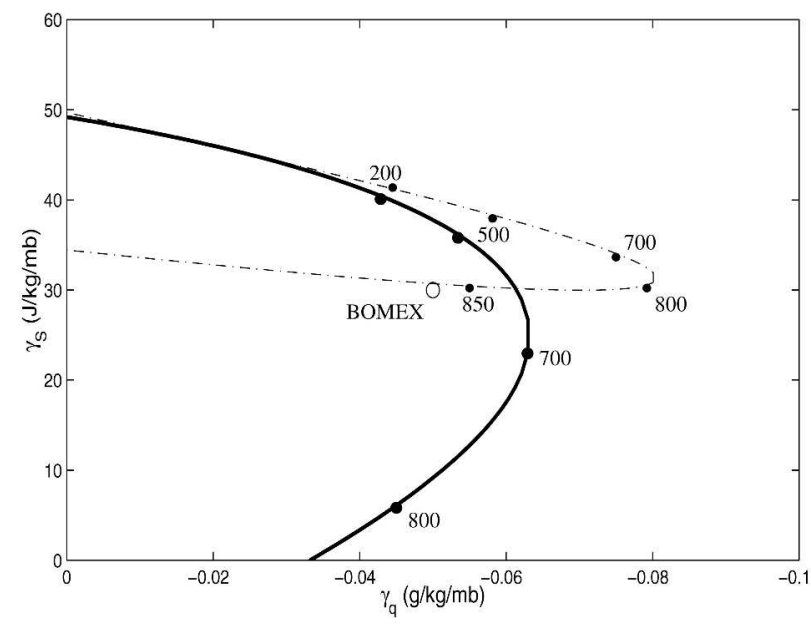

FIG. 4. Possible steady-state gradients $\left(\gamma_{q}, \gamma_{s}\right)$ with (solid line) BOMEX large-scale conditions and fluxes; (dash-dotted) BOMEX large-scale conditions and $\left(F_{\mathrm{cb}}^{q}, F_{\mathrm{cb}}^{s}\right)=\left(100 \mathrm{~W} \mathrm{~m}^{-2},-4\right.$ $\mathrm{W} \mathrm{^{-2 }}$ ); the labeled dots indicate the inversion height along the equilibrium line and the circle indicates the observed gradients. 
where $\bar{\omega}=(1-\lambda) \omega_{i+}+\lambda \omega_{\mathrm{ct}}$ is the equivalent subsidence at the inversion.

This simple model of the inversion gives an additional degree of freedom $\lambda$ to the system and allows the subsidence at the top of the inversion to be smaller than the subsidence at the top of the cloud, as has been observed (Holland and Rasmusson 1973; Augstein et al. 1973).

The two extreme terms of (34) lead to a cubic equation in the cloud depth $\Delta p$,

$$
\begin{aligned}
\frac{1}{2 \Delta p_{\mathrm{sc}}}\left(\frac{\Gamma_{q}}{\gamma_{q}}-\frac{\Gamma_{s}}{\gamma_{s}}\right) \Delta p^{3} & +\left[\frac{\Gamma_{q}}{\gamma_{q}}-\frac{\Gamma_{s}}{\gamma_{s}}+\frac{1}{2 \Delta p_{\mathrm{sc}}}\left(\frac{\Delta q_{i}^{o}}{\gamma_{q}}-\frac{\Delta s_{i}^{o}}{\gamma_{s}}\right)+\frac{R}{\omega_{\mathrm{cb}} \gamma_{s}}\left(\frac{\Gamma_{q}}{\gamma_{q}}-1\right)\right] \Delta p^{2} \\
+ & {\left[\frac{\Delta q_{i}^{o}}{\gamma_{q}}-\frac{\Delta s_{i}^{o}}{\gamma_{s}}+\frac{R}{\omega_{\mathrm{cb}} \gamma_{s}} \frac{\Delta q_{i}^{o}}{\gamma_{q}}+\frac{s_{\mathrm{cb}}^{e}}{\gamma_{s}}\left(\frac{\Gamma_{q}}{\gamma_{q}}-1\right)-\frac{q_{\mathrm{cb}}^{e}}{\gamma_{q}}\left(\frac{\Gamma_{s}}{\gamma_{s}}-1\right)\right] \Delta p+\frac{s_{\mathrm{cb}}^{e}}{\gamma_{s}} \frac{\Delta q_{i}^{o}}{\gamma_{q}}-\frac{q_{\mathrm{cb}}^{e}}{\gamma_{q}} \frac{\Delta s_{i}^{o}}{\gamma_{s}}=0 . }
\end{aligned}
$$

Figure 6 shows the most realistic $p_{\mathrm{ct}}=p_{\mathrm{cb}}-\Delta p$ (i.e., the closest to $850 \mathrm{mb}$ ) where $\Delta p$ is solution of Eq. (35) and the corresponding mixing-line parameter $\lambda$ computed with (34), using the large-scale conditions and parameters given in Tables 1 and 2. If the model can produce plausible altitudes for the inversion, those are underestimated. For the observed vertical gradients, the altitude of the inversion would be about $20 \mathrm{mb}$ below the observed altitude. Moreover, the mixing-line parameter $\lambda$ is supposed to be between 0 and 1 , which is not the case for most of the domain including the observed gradients. To the extent the BOMEX data are representative of a steady solution, this shows that a thin inversion following a mixing line is another incompatible idealization with the constant gradient hypothesis.

From these results it is unclear whether the source of the incompatibility is the bias on the fluxes at the cloud top that originates from the oversimplification of the profiles inside the cloud or if the inversion layer needs to be described by models that are more complex than a mixing line. In either case it is evident that the idealization of the profiles, either inside the cloud or in the inversion layer, more strongly constrains the evolution of the system than is generally appreciated. The fact that the constant gradient hypothesis is incompatible with the mass-flux scheme and both idealized models of the inversion points to this assumption as the main overconstraint. It thus seems interesting to study what equilibrium can be reached by releasing this constraint.

\section{Relaxed gradient laws}

In the previous section, we showed that the assumption of a constant gradient in the cloud layer places unrealistic constraints on the model of the turbulent fluxes that keeps the system from reaching an equilibrium. Thus, it makes sense to relax this assumption and study what steady solutions look like given a model of the mixing (e.g., Stevens 2000). Furthermore, we can investigate whether a change of the parameterization of turbulence allows a better representation of the inversion layer.

SH96 showed using a 1D model that a parameterization of the cloudy boundary layer with constant frac- tional entrainment and detrainment rates $\varepsilon$ and $\delta$ could reproduce the quasi-stationary state observed during BOMEX. The value of $\varepsilon$ and $\delta$ were inspired by a LES run (SC95). It is interesting to analyze the steady profiles obtained by this model, in order to understand the influence of the parameterization on the vertical gradients, and to explain why the parameterization gives satisfactory results in a $1 \mathrm{D}$ model and not in Albrecht's framework. Additionally, we can compute the profiles produced by Albrecht's parameterization of the turbulent fluxes, which corresponds to running the Albrecht model without the constant gradient hypothesis.

\section{a. General equations of the cloud layer}

Without the constant gradient approximation, Eq. (1) for the local equilibrium of a conserved variable can be rewritten

$$
\partial_{t} \phi=\omega \partial_{p^{\prime}} \phi-g \partial_{p^{\prime}} F^{\phi}+\sigma_{\phi} .
$$

With the mass flux parameterization [Eqs. (6)-(8)], it yields a differential equation in $\left(\phi^{c}-\phi\right)$ :

$$
\partial_{p^{\prime}}\left(\phi^{c}-\phi\right)+\left(\varepsilon-\delta \frac{g M}{\omega+g M}\right)\left(\phi^{c}-\phi\right)=\frac{\sigma_{\phi}}{\omega+g M},
$$

which can be solved semianalytically:

$$
\phi^{c}-\phi=\Delta \phi_{\mathrm{cb}} e^{-H\left(p^{\prime}\right)}+\sigma_{\phi} e^{-H\left(p^{\prime}\right)} I\left(p^{\prime}\right),
$$

where

$$
\begin{aligned}
H\left(p^{\prime}\right) & =\int_{0}^{p^{\prime}}\left(\epsilon-\delta \frac{g M}{\omega+g M}\right) d p \quad \text { and } \\
I\left(p^{\prime}\right) & =\int_{0}^{p^{\prime}} \frac{e^{H(p)}}{(\omega+g M)} d p
\end{aligned}
$$

are integration factors. The vertical gradient can thus be written

$$
\partial_{p^{\prime}} \phi=-\frac{1}{\omega+g M}\left[\sigma_{\phi}+\delta g M e^{-H\left(p^{\prime}\right)}\left(\Delta \phi_{\mathrm{cb}}+\sigma_{\phi} I\left(p^{\prime}\right)\right)\right] .
$$




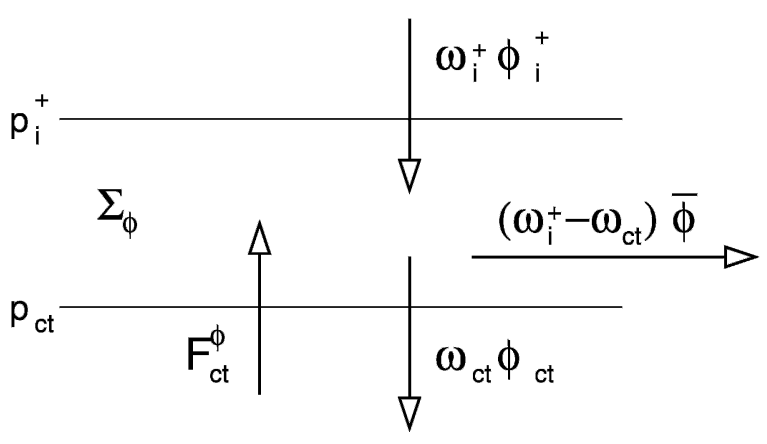

FIG. 5. Budget of the inversion layer for a conserved variable.

Equations (37) to (39) are valid whatever the profiles of $\omega, \epsilon$, and $\delta$. If these functions do not depend on the profiles, these equations can be integrated directly to compute the steady-state profiles, as we do in the next subsection.

The inversion will be modeled as described in section $4 \mathrm{~b}(2)$. The altitude of the inversion will be computed using the two extreme terms of Eq. (34), and the computation of $\lambda$ using the third term will be used as a test of the improvement of the representation of the inversion layer.

\section{b. Results for BOMEX case}

Figure 7 shows the steady-state profiles of the conserved variables and their gradients produced by the following models: (i) SH96's parameterization with $\epsilon=$ $2 \times 10^{-4} \mathrm{~Pa}^{-1}$ and $\delta=2.7 \times 10^{-4} \mathrm{~Pa}^{-1}$ (solid line), (ii) Albrecht's parameterization (with parameters from A84: $\tau_{a}=1 / 2$ day and $A=0.5$; dash-dotted line), and (iii) the functions $\epsilon$ and $\delta$ tabulated from SC95 (dashed line). The former actually mimics the LES run of SC95. As in the previous experiments, the cloud-base fluxes are prescribed and their values are indicated in Table 1. Here, we also prescribe the convective mass flux at cloud base: it is chosen to match the results of LES (SC95), $M_{\mathrm{cb}}=0.025 \mathrm{~kg} \mathrm{~m}^{-2} \mathrm{~s}^{-1}$.

All three profiles look close to linear, but the vertical gradient of liquid static energy actually changes significantly with height (up to an order of magnitude for the reconstruction of the LES run). This change is fundamental in determining the variation of both vertical advection and turbulent heating with altitude: the modification of the vertical transport, both the large-scale and the turbulent components, permits a steady-state solution that was not possible to reach with a constant gradient.

The variation of the vertical gradients with height, namely the second derivatives of the conserved variable profiles, has an impact on the turbulence that is unclear in mass-flux parameterizations, while it is an explicit term in $K$-diffusion schemes. Actually, the cloud - environment difference depends much more on the variations of the gradient with height than the profile does (see Fig. A1 in SH96). As the turbulent fluxes are proportional to this cloud - environment difference in the bulk mass-flux scheme, a good representation of the cloud environment is thus crucial to the representation of the turbulent mixing. The variation of the vertical gradients within the cloud layer is therefore crucial to the existence of an equilibrium.

Although the three models produce similar profiles of the conserved variables themselves, the gradient profiles are quite different. SH96's parameterization appears to be tuned to resemble the LES run in the middle of the cloud, but the LES produces gradients that are smaller at the cloud base and larger at the cloud top than the ensuing parameterization. This suggests that the cloud - environment differences at cloud top produced by the LES are likely to be quite different from the ones produced by the parameterization that mimics the LES.

The gradients produced by Albrecht's parameterization are smaller than the ones produced by SH96's pa-
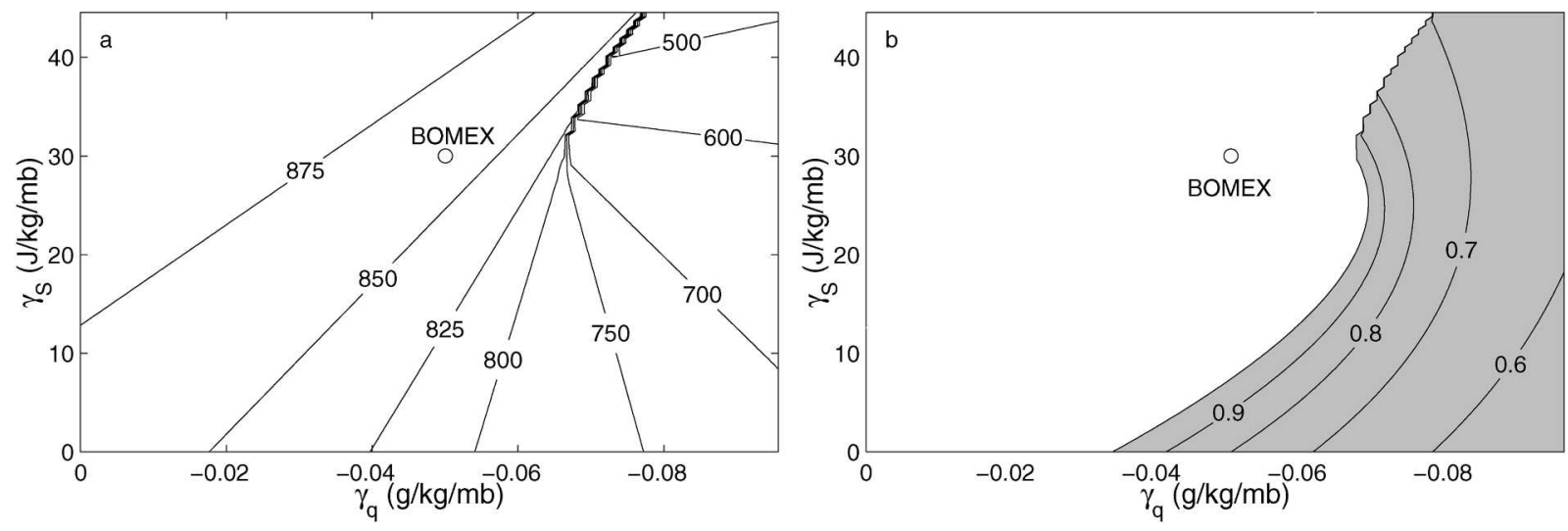

FIG. 6. (a) Altitude of the inversion (in mb) at the equilibrium, computed from (35), and (b) corresponding value of the mixing-line parameter $\lambda$; shaded: domain of possible equilibrium $(\lambda \varepsilon[0 ; 1])$. 

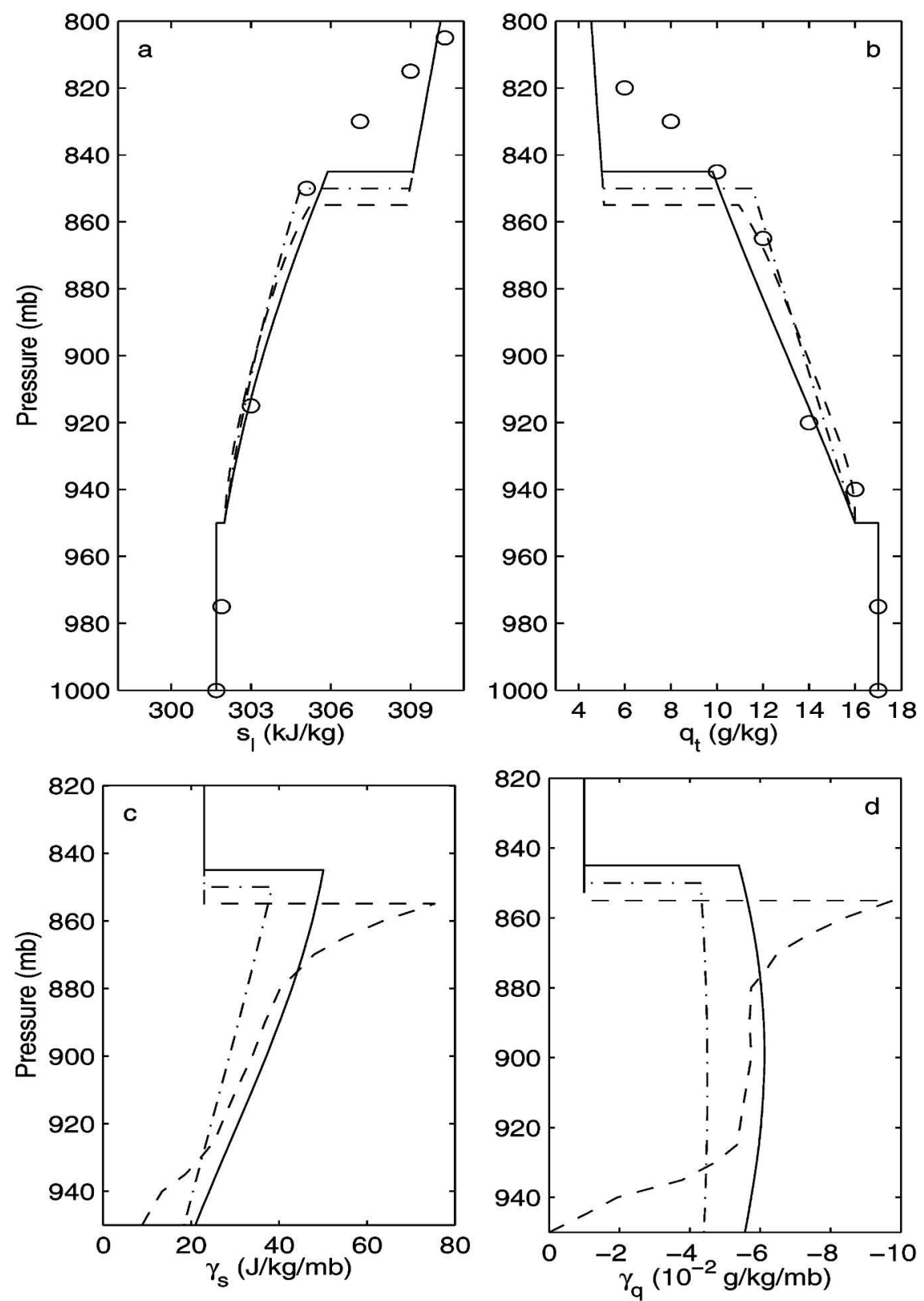

FIG. 7. Profiles of (a) static liquid energy, (b) total water, and (c), (d) their vertical gradients within the cloud layer, in BOMEX case; circles: observations; dashed: reconstruction of SC95's LES run; solid: SH96's parameterization; dash-dotted: Albrecht's parameterization.

rameterization, but their profiles show the same pattern: the liquid static energy gradient increases with height, while the total water gradient is more nearly constant. This similarity reflects the fact that Albrecht's parameterization is a linearized version of SH96's parameterization with modified parameters.

The turbulent flux profiles (see Fig. 8) are quite similar in the three different models except in the upper part of the cloud where they diverge. But the mass flux profiles exhibit more marked differences. Actually, the gap between the profiles of mass flux is compensated by the gap between the cloud - environment differences. This shows that, given a parameterization of the convective mass flux, the cloud - environment differences tend to adapt themselves to the mass flux in order to produce an appropriate turbulent flux profile. The cloud - environment differences have an important impact on the buoyancy profile, which might be problematic for buoyancy-based closures.

Although the models give different flux profiles at cloud top, all three models produce a realistic inversion around $850 \mathrm{mb}$, which tends to prove that the inversion 

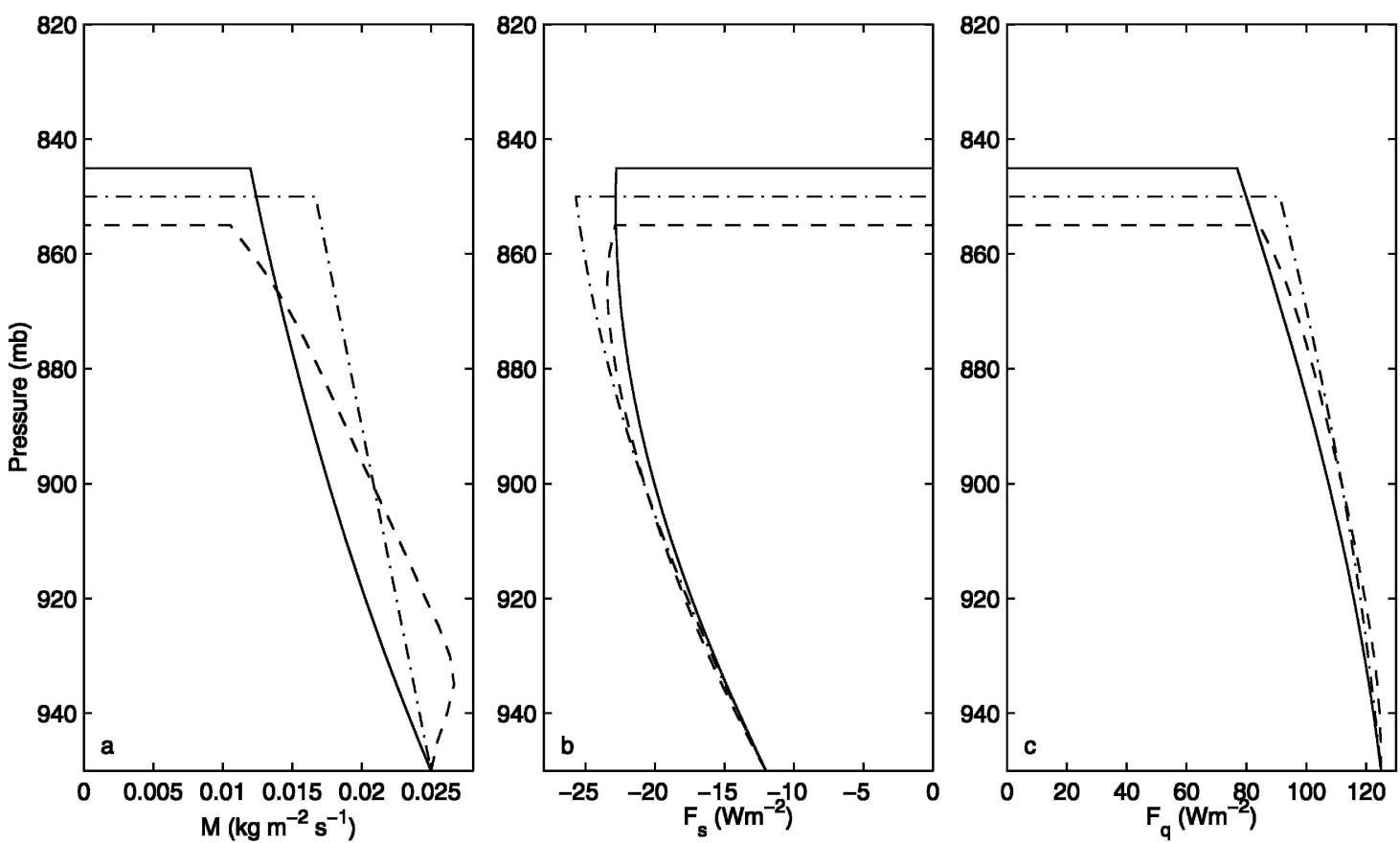

FIG. 8. Profiles of (a) convective mass flux, (b) turbulent heat flux, and (c) turbulent moisture flux in the cloud layer, in BOMEX case; dashed: reconstruction of SC95's LES run; solid: SH96's parameterization; dash-dotted: Albrecht's parameterization.

is well represented by the mixing line model used here; but the mixing line parameter is unrealistic in all three models (SH96's parameterization $\lambda=3.2$; Albrecht's parameterization $\lambda=2.7$; and reconstruction of SC95's LES run $\lambda=3.6$ ). The model of the inversion layer still seems oversimplified. Although this issue can be avoided by relaxing the constraint on cloud-base fluxes (Bretherton 2004, unpublished manuscript; see also Fig. 4 ), the $\lambda$ computed in our experiments are quite large compared to the realistic range [0;1], and the fluxes required to obtain a realistic $\lambda$ are expected to be significantly different from the LES results.

\section{Summary and discussion}

Our work showed that it is not possible to represent some observed situations with a constant gradient model when implemented with a bulk mass-flux parameterization. This framework can produce a limited domain of cases - most of which exhibit a convective mass flux that unrealistically increases with height. The change of the vertical gradient with height seems to be crucial to the existence of a steady state. SH96's parameterization of the mixing, as well as Albrecht's, allows the cloud layer to reach an equilibrium, mainly by increasing the vertical gradient of dry static energy with height.

The ability of different parameterizations to simulate realistic profiles of the conserved variables and their fluxes suggests that those profiles are not sufficient to validate a parameterization. The profiles of the gradient and cloud - environment differences appear as a better test. Unfortunately, they are difficult to observe directly, although LES may provide useful information in this respect.

In the absence of diabatic sources, the approximation of an infinitely thin inversion layer is also shown to overconstrain the system. Modeling the inversion as a thin layer following a mixing line allows one to simulate well the altitude of the inversion, with cloud-layer profiles that are either linear or produced by Albrecht's or SH96's parameterizations. But, in all cases, the thermodynamic structure of the inversion is unrealistic. This is problematic because the vertical structure at the top of the cloud layer and in the inversion is crucial in determining the presence or absence of stratiform cloudiness whose radiative forcing is important.

Of course, the constraints resulting from the constant gradient hypothesis or from these parameterizations, which are tuned to represent well the middle of the cloud, leads to a loose representation of the conserved variables and their fluxes at the top of the cloud. But, it seems that the main reason of the misrepresentation of the inversion is the fact that the processes at play in the inversion layer need to be addressed more accurately. Some of the processes are not well understoodpossible decrease of the subsidence speed with height, radiative cooling at the top of the cloud; but most of them are actually the same as in the cloud layer. Thus, it seems natural to take a unified approach, considering 
a unique layer extending from the cloud base to the top of the inversion.

So far, no consistent theory including the inversion layer has been developed: most of the parameterizations strive to reproduce the profiles within the cloud layer, and the inversion layer is considered a buffer that links the cloud layer to the free troposphere. A unified approach would require the parameterizations of the mixing to produce, at the top of the inversion, turbulent fluxes that vanish and profiles of the conserved variables that merge with the free-tropospheric profiles. The challenge in the construction of such a bulk model is to establish plausible hypotheses on the profiles of the conserved and their fluxes that (i) are of the same order of complexity, (ii) are consistent within the cloud layer, and (iii) meet the boundary conditions at the top of the inversion.

Last, one can only speculate how the vertical resolution of the GCMs can affect the parameterization of the shallow convection. In general, the parameterization of the CTBL is very sensitive to this resolution (Lenderink et al. 2005). Our work also suggests that some precision in the vertical profiles of the thermodynamic variables is needed to obtain a good representation of the shallow convection. Low vertical resolutions in climate versions of the GCMs give a representation of the vertical profiles that are hardly better than a linear profile, and the inversion layer is not well represented either. In this case, the parameterization of the shallow convection is unlikely to produce realistic profiles or cloud fields, thus imperiling attempts to rationalize the role of clouds in climate change.

Acknowledgments. The authors thank Sylvain Cheinet for many useful discussions. Bjorn Stevens' work on this project was supported by NSF Grant ATM9985413. Gilles Bellon was partly sponsored by NSF Grant ATM00-82131.

\section{APPENDIX A}

\section{A Derivation of the Domain of Possible Equilibrium for the Relaxed Flux Laws}

Replacing Eqs. (7) and (8) in the derivative of Eq. (8) gives

$$
\begin{aligned}
\partial_{p^{\prime}} F^{\phi} & =\partial_{p^{\prime}} M\left(\phi^{c}-\phi\right)+M\left(\partial_{p^{\prime}} \phi^{c}-\gamma_{\phi}\right) \\
& =(\epsilon-\delta) M\left(\phi^{c}-\phi\right)+M\left[-\epsilon\left(\phi^{c}-\phi\right)-\gamma_{\phi}\right] \\
& =-\delta F^{\phi}-M \gamma_{\phi} .
\end{aligned}
$$

Using this equation for $s_{l}$ and $q_{t}$ yields a linear system in $M$ and $\delta$ that allows us to express the functions $M\left(p^{\prime}\right)$ and $\delta\left(p^{\prime}\right)$ as functions of the turbulent fluxes, their derivatives and the vertical gradients [Eqs. (21) and (22)].

Furthermore, Eq. (7) can be rewritten

$$
\epsilon-\delta=\frac{\partial_{p^{\prime}} M}{M}=\partial_{p^{\prime}}(\ln (M))
$$

which yields

$$
\begin{aligned}
& \epsilon-\delta=\partial_{p^{\prime}} \ln \left(\frac{\partial_{p^{\prime}} F^{q}}{F^{q}}-\frac{\partial_{p^{\prime}} F^{s}}{F^{s}}\right)-\partial_{p^{\prime}} \ln \left(-\frac{\gamma_{q}}{F^{q}}+\frac{\gamma_{s}}{F^{s}}\right) \\
& =\frac{\frac{\partial_{p^{\prime} p^{\prime}} F^{q}}{F^{q}}-\frac{\partial_{p^{\prime} p^{\prime}} F^{s}}{F^{s}}}{\frac{\partial_{p^{\prime}} F^{q}}{F^{q}}-\frac{\partial_{p^{\prime}} F^{s}}{F^{s}}}-\frac{\frac{\partial_{p^{\prime}} F^{q}}{\gamma_{q}}-\frac{\partial_{p^{\prime}} F^{s}}{\gamma_{s}}}{\frac{F^{q}}{\gamma_{q}}-\frac{F^{s}}{\gamma_{s}}} .
\end{aligned}
$$

Replacing the expression of $\delta$ by the previous result [Eq. (22)] gives the expression of $\epsilon$ [Eq. (23)].

The conditions of sign on $M$ and $\delta$ can be turned into conditions on the ratios of the fluxes, their derivatives, and the gradients of conserved variables, using their physically consistent signs $\left(F^{q}>0, \gamma_{q}<0, F^{s}<0\right.$, and $\left.\gamma_{s}>0\right)$ :

$$
\begin{aligned}
M>0 \Leftrightarrow & \left(\frac{\partial_{p^{\prime}} F^{q}}{F^{q}}<\frac{\partial_{p^{\prime}} F^{s}}{F^{s}} \text { and } \frac{F^{q}}{\gamma_{q}}<\frac{F^{s}}{\gamma_{s}}\right) \text { or } \\
& \left(\frac{\partial_{p^{\prime}} F^{q}}{F^{q}}>\frac{\partial_{p^{\prime}} F^{s}}{F^{s}} \text { and } \frac{F^{q}}{\gamma_{q}}>\frac{F^{s}}{\gamma_{s}}\right), \\
\delta>0 \Leftrightarrow & \left(\frac{\partial_{p^{\prime}} F^{q}}{\gamma_{q}}>\frac{\partial_{p^{\prime}} F^{s}}{\gamma_{s}} \text { and } \frac{F^{q}}{\gamma_{q}}<\frac{F^{s}}{\gamma_{s}}\right) \text { or } \\
& \left(\frac{\partial_{p^{\prime}} F^{q}}{\gamma_{q}}<\frac{\partial_{p^{\prime}} F^{s}}{\gamma_{s}} \text { and } \frac{F^{q}}{\gamma_{q}}>\frac{F^{s}}{\gamma_{s}}\right) .
\end{aligned}
$$

Using the expression of the derivative of fluxes and the fact that the radiative rate is negative $(R<0)$, we can discriminate one of the conditions:

$$
\frac{\partial_{p^{\prime}} F^{q}}{\gamma_{q}}=\frac{\omega}{g}>\frac{\omega}{g}+\frac{R}{g \gamma_{s}}=\frac{\partial_{p^{\prime}} F^{s}}{\gamma_{s}} .
$$

Consequently, considering the signs of $\gamma_{\phi}$ and $F^{\phi}$, the conditions (A3) and (A4) combine into

$$
M>0 \text { and } \delta>0 \Leftrightarrow \frac{F^{q}}{\gamma_{q}}<\frac{F^{s}}{\gamma_{s}} \text { and } \frac{\partial_{p^{\prime}} F^{q}}{F^{q}}<\frac{\partial_{p^{\prime}} F^{s}}{F^{s}} .
$$

We will refer to the first condition of the rhs as $\mathrm{C} 1$ and the second as $\mathrm{C} 2$. Conditions $\mathrm{C} 1$ and $\mathrm{C} 2$ imply $\partial_{p^{\prime}} F^{q} / \gamma_{q}$ $>\partial_{p^{\prime}} F^{s} / \gamma_{s}$, which can be written $\partial_{p^{\prime}}\left(F^{q} / \gamma_{q}-F^{s} / \gamma_{s}\right)>0$. Therefore, $F^{q} / \gamma_{q}-F^{s} / \gamma_{s}$ increases with $p^{\prime}$ and will be negative over the whole cloud layer if it is negative at cloud top. Thus $\mathrm{C} 1$ is equivalent to

$$
\frac{F_{\mathrm{ct}}^{q}}{\gamma_{q}}-\frac{F_{\mathrm{ct}}^{s}}{\gamma_{s}}<0
$$

Using the equilibrium flux profiles [Eq. (5)], we can further write $\mathrm{C} 1$ as follows: 


$$
\frac{F_{\mathrm{cb}}^{q}}{\gamma_{q}}-\frac{F_{\mathrm{cb}}^{s}+R \Delta p / g}{\gamma_{s}}<0,
$$

which leads to the lhs in inequality (24).

Furthermore, considering the opposite signs of the fluxes, $\mathrm{C} 2$ is equivalent to $F^{s} \partial p^{\prime} F^{q}-F^{q} \partial p^{\prime} F^{s}>0$. Besides, we note that, according to the equilibrium flux profiles and their derivatives [Eqs. (5) and (4)], the second derivative of the flux is expressed as

$$
\partial_{p^{\prime} p^{\prime}} F^{\phi}=\frac{D}{g} \gamma_{\phi} .
$$

Thus, considering the opposite signs of the gradients and C1,

$$
\partial_{p^{\prime}}\left(F^{s} \partial_{p^{\prime}} F^{q}-F^{q} \partial_{p^{\prime}} F^{s}\right)=-\frac{D}{g} \gamma_{q} \gamma_{s}\left(\frac{F^{q}}{\gamma_{q}}-\frac{F^{s}}{\gamma_{s}}\right)<0 ;
$$

thus, $F^{s} \partial_{p^{\prime}} F^{q}-F^{q} \partial_{p^{\prime}} F^{s}$ decreases with $p^{\prime}$, and will be positive over the whole cloud layer if it is positive at cloud top. Thus $\mathrm{C} 2$ is equivalent to

$$
F_{\mathrm{ct}}^{s} \partial_{p^{\prime}} F^{q}(\Delta p)-F_{\mathrm{ct}}^{q} \partial_{p^{\prime}} F^{s}(\Delta p)>0 .
$$

Using the equilibrium flux profiles and its derivative [Eqs. (5) and (4)], we can further write $\mathrm{C} 2$ as follows:

$\frac{F_{\mathrm{cb}}^{q}}{\gamma_{q}}\left(1+\frac{R}{\omega_{\mathrm{ct}} \gamma_{s}}\right)-\frac{F_{\mathrm{cb}}^{s}}{\gamma_{s}}-\frac{R}{g \gamma_{s}} \frac{\Delta p^{2}}{2\left(\Delta p+\Delta p_{\mathrm{sc}}\right)}>0$,

which leads to the rhs in inequality (24).

The second term of the rhs of (23) is actually twice $\delta$. Therefore, we can write $\epsilon=h\left(p^{\prime}, 2\right)$ and $\epsilon-\delta=$ $h\left(p^{\prime}, 1\right)$ with

$$
\begin{aligned}
h\left(p^{\prime}, n\right)= & -\frac{D}{g} \frac{\gamma_{q} \gamma_{s}}{F^{q} F^{s}} \frac{F^{q} / \gamma_{q}-F^{s} / \gamma_{s}}{\partial_{p^{\prime}} F^{q} / F^{q}-\partial_{p^{\prime}} F^{s} / F^{s}} \\
& +n \frac{R / g \gamma_{s}}{F^{q} / \gamma_{q}-F^{s} / \gamma_{s}},
\end{aligned}
$$

where the second derivatives of the equilibrium flux profiles have been replaced by their expression (A9), as well as the first derivatives in the second term:

$$
\frac{\partial_{p^{\prime}} F^{q}}{\gamma_{q}}-\frac{\partial_{p^{\prime}} F^{s}}{\gamma_{s}}=-\frac{R}{g \gamma_{s}} .
$$

The conditions on the signs of $\epsilon$ and $\epsilon-\delta$ can thus be assessed by studying the sign of $h$. Using the conditions derived from $M>0$ and $\delta>0$, we can write

$$
\begin{aligned}
h>0 \Leftrightarrow H\left(p^{\prime}, n\right)= & \left(\frac{F^{q}}{\gamma_{q}}-\frac{F^{s}}{\gamma_{s}}\right)^{2} \\
& -n \frac{R}{D \gamma_{s}}\left(\frac{F^{s}}{\gamma_{s}} \frac{\partial_{p^{\prime}} F^{q}}{\gamma_{q}}-\frac{F^{q}}{\gamma_{q}} \frac{\partial_{p^{\prime}} F^{s}}{\gamma_{s}}\right)<0 .
\end{aligned}
$$

We derive $H$ to assess its variation with $p^{\prime}$ :

$$
\begin{aligned}
\partial_{p^{\prime}} H= & 2\left(\frac{\partial_{p^{\prime}} F^{q}}{\gamma_{q}}-\frac{\partial_{p^{\prime}} F^{s}}{\gamma_{s}}\right)\left(\frac{F^{q}}{\gamma_{q}}-\frac{F^{s}}{\gamma_{s}}\right) \\
& -n \frac{R}{D \gamma_{s}}\left(\frac{F^{s}}{\gamma_{s}} \frac{\partial_{p^{\prime} p^{\prime}} F^{q}}{\gamma_{q}}-\frac{F^{q}}{\gamma_{q}} \frac{\partial_{p^{\prime} p^{\prime}} F^{s}}{\gamma_{s}}\right) \\
= & (n-2) \frac{R}{g \gamma_{s}}\left(\frac{F^{q}}{\gamma_{q}}-\frac{F^{s}}{\gamma_{s}}\right) .
\end{aligned}
$$

So, for $n=2, H$ is independent of $p^{\prime}$; therefore, the condition $\epsilon>0$ can be computed anywhere within the cloud. We will compute it at the cloud base $(H(0,2)<$ $0)$. For $n=1, H$ decreases with $p^{\prime}$. The condition for increasing mass flux $(\epsilon-\delta>0)$ for the whole cloud layer is thus equivalent to $H(0,1)<0$, and the condition for a strictly decreasing mass flux is equivalent to $H(\Delta p, 1)>0$. Here $H$ is quadratic in $\gamma_{q}$ and $\gamma_{s}$ and can be rewritten, replacing the fluxes and their derivatives by their equilibrium profiles (5) and (4):

$$
\begin{aligned}
H\left(p^{\prime}, n\right)= & \frac{F_{\mathrm{cb}}^{q 2}}{\gamma_{q}^{2} \gamma_{s}^{2}}\left\{\gamma_{s}^{2}+\gamma_{s} \frac{\gamma_{q}}{F_{\mathrm{cb}}^{q}}\left[n \frac{R}{g}\left(\Delta p_{\mathrm{sc}}+p^{\prime}\right)-2\left(F_{\mathrm{cb}}^{s}+\frac{R}{g} p^{\prime}\right)\right]\right. \\
& \left.+\frac{\gamma_{q}^{2}}{F_{\mathrm{cb}}^{q 2}}\left[\left(F_{\mathrm{cb}}^{s}+\frac{R}{g} p^{\prime}\right)^{2}-n \frac{R}{g}\left(\left(F_{\mathrm{cb}}^{s}+\frac{R}{g} p^{\prime}\right)\left(\Delta p_{\mathrm{sc}}+p^{\prime}\right)-\frac{R}{D}\left(\frac{F_{\mathrm{cb}}^{q}}{\gamma_{q}}+\frac{D D_{2}\left(p^{\prime}\right)}{2 g}\right)\right)\right]\right\},
\end{aligned}
$$

with $D_{2}\left(p^{\prime}\right)=\left(\Delta p_{\mathrm{sc}}+p^{\prime}\right)^{2}-\Delta p_{\mathrm{sc}}^{2}$. Here $H$ is negative for $\gamma_{s}$ between the roots $\gamma_{s}^{-}$and $\gamma_{s}^{+}$of this quadratic equation, and positive elsewhere. The roots can be ex- pressed as a function of $\gamma_{q}$, the cloud-base fluxes, and the large-scale forcing:

$$
\gamma_{s}^{ \pm}=\frac{\gamma_{q}}{F_{\mathrm{cb}}^{q}}\left[F_{\mathrm{cb}}^{s}+\frac{R}{g} p^{\prime}-\frac{n R}{2 g}\left(\Delta p_{\mathrm{sc}}+p^{\prime}\right)\left(1 \mp \sqrt{1-\frac{4 \frac{g F_{\mathrm{cb}}^{q}}{D \gamma_{q}}+2 D_{2}\left(p^{\prime}\right)}{n\left(\Delta p_{\mathrm{sc}}+p^{\prime}\right)^{2}}}\right)\right]
$$


For the parameter range of interest, $\gamma_{s}^{-}$is negative. The cloud layer is only conditionally unstable, so $\gamma_{s}$ is positive, and we will focus on its position with respect to $\gamma_{s}^{+}$.
We can deduce the three following conditions, which are reproduced in a more concise form in Eqs. (25), (27), and (28):

$$
\begin{gathered}
\epsilon>0 \Leftrightarrow \gamma_{s}<\frac{\gamma_{q}}{F_{\mathrm{cb}}^{q}}\left[F_{\mathrm{cb}}^{s}+\frac{R}{g} \Delta p_{\mathrm{sc}}\left(\sqrt{1-2 \frac{g F_{\mathrm{cb}}^{q}}{D \gamma_{q} \Delta p_{\mathrm{sc}}^{2}}}-1\right)\right], \\
\epsilon-\delta>0 \Leftrightarrow \gamma_{s}<\frac{\gamma_{q}}{F_{\mathrm{cb}}^{q}}\left[F_{\mathrm{cb}}^{s}+\frac{R}{2 g} \Delta p_{\mathrm{sc}}\left(\sqrt{1-4 \frac{g F_{\mathrm{cb}}^{q}}{D \gamma_{q} \Delta p_{\mathrm{sc}}^{2}}}-1\right)\right], \\
\epsilon-\delta<0 \Leftrightarrow \gamma_{s}>\frac{\gamma_{q}}{F_{\mathrm{cb}}^{q}}\left[F_{\mathrm{cb}}^{s}+\frac{R}{2 g} \Delta p_{\mathrm{sc}}\left(\frac{\Delta p}{\Delta p_{\mathrm{sc}}}-1+\sqrt{1-2 \frac{\Delta p}{\Delta p_{\mathrm{sc}}}-\frac{\Delta p^{2}}{\Delta p_{\mathrm{sc}}^{2}}-4 \frac{g F_{\mathrm{cb}}^{q}}{D \gamma_{q} \Delta p_{\mathrm{sc}}^{2}}}\right)\right] .
\end{gathered}
$$

\section{APPENDIX B}

\section{Summary of Symbols}

$$
\begin{aligned}
& p^{\prime} \equiv p_{\mathrm{cb}}-p \\
& \Delta p_{\mathrm{sc}} \\
& \Delta p \\
& \pi \equiv \Delta p / \Delta p_{\mathrm{sc}}
\end{aligned}
$$$$
\text { a. Vertical coordinates }
$$

\section{b. Subscripts}

0

$$
\mathrm{cb}
$$

A

ct

$i^{+}$

\section{c. Conserved variable profiles}

$\phi$

$\Delta \phi_{0}$
$\gamma_{\phi}$
$\phi_{i}^{o}$
$\Delta \phi_{i}^{o} \equiv \phi_{i}^{o}-\phi_{0}-\Delta \phi_{0}$
$\Gamma_{\phi}$
$\bar{\phi} \equiv \lambda \phi_{i+}+(1-\lambda) \phi_{\mathrm{ct}}$
$\phi^{c}$
$\Delta \phi_{\mathrm{cb}}$

Subcloud

Cloud base

Middle of the cloud

Cloud top

Top of the inversion

Conserved variable, either $s_{l}$ or $q_{t}$. The subscripts $l$ and $t$ are omitted when $\phi$ has another subscript or when $\phi$ itself is a subscript or superscript; $\phi$ is used for the clearsky profile in the cloud layer. Jump at cloud base Gradient of $\phi$ in the cloud layer

Reference free-tropospheric profile $\phi$ at cloud base Reference free-troposphere/ boundary layer difference at cloud base Free-tropospheric gradient of $\phi$

Divergence-averaged $\phi$ of the inversion layer

Cloud profile Cloud - environment difference at cloud base

$$
d \equiv\left(\phi^{c}-\phi\right) / \Delta \phi_{\mathrm{cb}}
$$$$
\zeta_{\phi} \equiv \gamma_{\phi} / \Delta \phi_{\mathrm{cb}}
$$

\section{d. Large-scale forcing}

$\omega$

$D$

$\bar{\omega} \equiv(1-\lambda) \omega_{i+}+\lambda \omega_{\mathrm{ct}}$

$\sigma_{\phi}$

$R \equiv \sigma_{s l}$

$\Sigma_{\phi}$

$\Delta F^{R} \equiv-\Sigma_{s l}$

e. Turbulent mixing

$F^{\phi}$

$M$

$f \equiv \mathrm{M} / M_{\mathrm{cb}}$

$\epsilon$

$\delta$

\section{f. Miscellaneous}

$\alpha$

$\beta_{F}$

$q_{\mathrm{cb}}^{e}$

$s_{\mathrm{cb}}^{e}$
Normalized cloud - environment difference

Ratio of the stratification to the cloud-base difference

\section{Vertical speed}

Rate of divergence

Equivalent subsidence at the inversion

Adiabatic source of $\phi$

Radiative cooling

Adiabiatic source integrated over the inversion layer

Jump of the upward net radiative flux at the inversion

Upward turbulent flux of $\phi$ Convective mass flux

Normalized convective mass flux

Entrainment rate

Detrainment rate

Ratio of the cooling of the subcloud layer to the warming of this layer due to the turbulent flux at cloud base Bowen ratio at cloud base Equivalent humidity of the turbulent water flux at cloud base

Equivalent energy of the turbulent sensible flux at cloud base 


\section{REFERENCES}

Albrecht, B. A., 1984: A model study of downstream variations of the thermodynamic structure of the trade winds. Tellus, $\mathbf{3 6 A}$, 187-202.

—, A. K. Betts, W. H. Schubert, and S. K. Cox, 1979: A model of the thermodynamic structure of the trade-wind boundary layer: Part I. Theoretical formulation and sensitivity tests. J. Atmos. Sci., 36, 73-89.

Augstein, E., H. Riehl, F. Ostapoff, and V. Wagner, 1973: Mass and energy transports in an undisturbed Atlantic trade-wind flow. Mon. Wea. Rev., 101, 101-111.

Betts, A. K., and W. Ridgway, 1988: Coupling of the radiative, convective, and surface fluxes over the equatorial Pacific. $J$. Atmos. Sci., 45, 522-546.

— convective boundary layer over a tropical ocean. J. Atmos. Sci., 46, 2621-2641.

Blyth, A. M., 1993: Entrainment in cumulus clouds. J. Appl. Meteor., 32, 626-641.

Bony, S., J.-L. Dufresne, H. Le Treut, J.-J. Morcrette, and C. Senior, 2004: On dynamic and thermodynamic components of cloud changes. Climate Dyn., 22, 71-86.

Bretherton, C. S., 1993: Understanding Albrecht's model of trade cumulus cloud fields. J. Atmos. Sci., 50, 2264-2283.

Cess, R. D., and Coauthors, 1996: Cloud feedback in atmospheric general circulation models: An update. J. Geophys. Res., 101, 12 791-12 794

Holland, J. Z., and E. M. Rasmusson, 1973: Measurements of the atmospheric mass, energy, and momentum budgets over a 500-kilometer square of tropical ocean. Mon. Wea. Rev., 101, 44-55.

Lenderink, G., and Coauthors, 2005: The diurnal cycle of shallow cumulus clouds over land: A single column model intercomparison study. Quart. J. Roy. Meteor. Soc., in press.

Lilly, D. K., 1968: Models of cloud-topped mixed layers under a strong inversion. Quart. J. Roy. Meteor. Soc., 94, 292-309.

Miller, R. L., 1997: Tropical thermostats and low cloud cover. J. Climate, 10, 409-440.

Siebesma, A. P., and J. W. M. Cuijpers, 1995: Evaluation of parametric assumptions for shallow cumulus convection. J. Atmos. Sci., 52, 650-666.

- and A. A. M. Holtslag, 1996: Model impacts of entrainment and detrainment rates in shallow cumulus convection. J. Atmos. Sci., 53, 2354-2364.

Stevens, B., 2000: Quasi-steady analysis of a PBL model with an eddy-diffusivity profile and nonlocal fluxes. Mon. Wea. Rev., 128, 824-836.

- and Coauthors, 2001: Simulations of trade wind cumuli under a strong inversion. J. Atmos. Sci., 58, 1870-1891.

Tiedtke, M., 1989: A comprehensive mass flux scheme for cumulus parameterization in large-scale models. Mon. Wea. Rev., 117, $1779-1800$ 